\title{
Best Proximity Points of Generalized Semicyclic Impulsive Self-Mappings: Applications to Impulsive Differential and Difference Equations
}

\author{
M. De la Sen ${ }^{1}$ and E. Karapinar ${ }^{2}$ \\ ${ }^{1}$ Institute of Research and Development of Processes, University of Basque Country, Campus of Leioa (Bizkaia), \\ P.O. Box 644, 48940 Bilbao, Spain \\ ${ }^{2}$ Department of Mathematics, ATILIM University, Incek 06586, Ankara, Turkey
}

Correspondence should be addressed to M. De la Sen; manuel.delasen@ehu.es

Received 3 May 2013; Accepted 1 August 2013

Academic Editor: Calogero Vetro

Copyright (C 2013 M. De la Sen and E. Karapinar. This is an open access article distributed under the Creative Commons Attribution License, which permits unrestricted use, distribution, and reproduction in any medium, provided the original work is properly cited.

\begin{abstract}
This paper is devoted to the study of convergence properties of distances between points and the existence and uniqueness of best proximity and fixed points of the so-called semicyclic impulsive self-mappings on the union of a number of nonempty subsets in metric spaces. The convergences of distances between consecutive iterated points are studied in metric spaces, while those associated with convergence to best proximity points are set in uniformly convex Banach spaces which are simultaneously complete metric spaces. The concept of semicyclic self-mappings generalizes the well-known one of cyclic ones in the sense that the iterated sequences built through such mappings are allowed to have images located in the same subset as their pre-image. The self-mappings under study might be in the most general case impulsive in the sense that they are composite mappings consisting of two selfmappings, and one of them is eventually discontinuous. Thus, the developed formalism can be applied to the study of stability of a class of impulsive differential equations and that of their discrete counterparts. Some application examples to impulsive differential equations are also given.
\end{abstract}

\section{Introduction}

Fixed point theory has an increasing interest in research in the last years especially because of its high richness in bringing together several fields of Mathematics including classical and functional analysis, topology, and geometry [18]. There are many fields for the potential application of this rich theory in Physics, Chemistry, and Engineering, for instance, because of its usefulness for the study of existence, uniqueness, and stability of the equilibrium points and for the study of the convergence of state-solution trajectories of differential/difference equations and continuous, discrete, hybrid, and fuzzy dynamic systems as well as the study of the convergence of iterates associated to the solutions. A basic key point in this context is that fixed points are equilibrium points of solutions of most of many of the above problems. Fixed point theory has also been investigated in the context of the so-called cyclic self-mappings [8-20] and multivalued mappings [21-32]. One of the relevant problems under study in fixed point theory is that associated with $p$ cyclic mappings which are defined on the union of a number of nonempty subsets $A_{i} \subset X ; \forall i \in \bar{p}=\{1,2, \ldots, p\}$ of metric $(X, d)$ or Banach spaces $(X,\|\|)$. There is an exhaustive background literature concerning nonexpansive, nonspreading, and contractive $p$-cyclic self-mappings $T: \bigcup_{i \in \bar{p}} A_{i} \rightarrow$ $\bigcup_{i \in \bar{p}} A_{i}$, for example, [8-20], including rational contractivetype conditions and [20,33], and references therein, and for various kinds of multivalued mappings. See, for instance [21-32] and references therein. A key point in the study of contractive cyclic self-mappings is that if the subsets $A_{i}$ for $i \in \bar{p}$ are disjoint then the convergence of the sequence of iterates $x_{n+1}=T x_{n} ; \forall n \in \mathbf{Z}_{0+}\left(\mathbf{Z}_{0+}=\mathbf{Z}_{+} \cup\{0\}\right), x_{0} \in$ $\bigcup_{i \in \bar{p}} A_{i}$, is only possible to best proximity points. The existence of such fixed points, its uniqueness and associated 
properties are studied rigorously in [11-13] in the framework of uniformly convex metric spaces, in [14-17], and in [12, 19] for Meir-Keeler type contractive cyclic self-mappings. In this paper, we introduce the notions of nonexpansive and contractive $p$-semicyclic impulsive self-mappings and investigate the best proximity and fixed points of those maps. The properties of boundedness and convergence of distances are studied in metric spaces, while those of the iterated sequences $x_{n+1}=T x_{n} ; \forall n \in \mathbf{Z}_{0+}, x_{0} \in \bigcup_{i \in \bar{p}} A_{i}$, are studied in uniformly convex Banach spaces. It is also seen through examples that the above combined constraint for distances is relevant for the description of the solutions of impulsive differential equations and discrete impulsive equations and for associate dynamic systems. The boundedness of the sequences of distances between consecutive iterates is guaranteed for nonexpansive $p$-semicyclic self-mappings while its convergence is proved for asymptotically contractive $p$-semicyclic self-mappings. In this case, the existence of a limit set for such sequences is proved. Such a limit set contains best proximity points if the asymptotically contractive $p$ semicyclic self-mapping is asymptotically $p$-cyclic, $(X, d)$ is a complete metric space which is also a uniformly convex Banach space $(X,\|\|)$, and the subsets $A_{i} \subset X ; \forall i \in \bar{p}$ are nonempty, closed, and convex. It has to be pointed out that the standard nonexpansive and contractive cyclic selfmappings may be viewed as a particular case of those proposed in this paper since it suffices to define the map so that any point of a subset is mapped in one of the adjacent subsets in the cyclic disposal and to define the second selfmapping of the composite impulsive one as identity.

\section{Nonexpansive and Contractive $p$-Semicyclic and $p$-Cyclic Impulsive Self-Mappings}

Consider a metric space $(X, d)$ and a composite self-mapping $T: \bigcup_{i \in \bar{p}} A_{i} \rightarrow \bigcup_{i \in \bar{p}} A_{i}$ of the form $T=T^{+} T^{-}$, where $A_{i}, i \in$ $\bar{p}$ are $p(\geq 2)$ nonempty closed subsets of $X$ with $A_{n p+i} \equiv A_{i}$; $\forall i \in \bar{p}, \forall n \in \mathbf{Z}_{0+}$ (in particular, $A_{p+1} \equiv A_{1}$ ) having a distance $D_{i}=d\left(A_{i}, A_{i+1}\right) \geq 0$ between any two adjacent subsets $A_{i}$ and $A_{i+1}$ of $X ; \forall i \in \bar{p}$. In order to facilitate the reading of the subsequent formal results obtained in the paper, it is assumed that $D=D_{i} ; \forall i \in \bar{p}$. Some useful types of such composite self-mappings for applications together with some of their properties in metric spaces are studied in this paper according to the following definition and its subsequent extensions.

Definition 1. The composite self-mapping $T\left(\equiv T^{+} T^{-}\right)$: $\bigcup_{i \in \bar{p}} A_{i} \rightarrow \bigcup_{i \in \bar{p}} A_{i}$ is said to be a $p$-semicyclic impulsive selfmapping if the following conditions hold:

(1) $T^{-}: \bigcup_{i \in \bar{p}} A_{i} \rightarrow \bigcup_{i \in \bar{p}} A_{i}$ is such that $T^{-} A_{i} \subseteq A_{i} \cup$ $A_{i+1} ; \forall i \in \bar{p}$ satisfies the constraint $d\left(T^{-} x, T^{-} y\right) \leq$ $K d(x, y)+(1-K) D ; \forall x \in A_{i}, \forall y \in A_{i+1}$, and $\forall i \in \bar{p}$ for some real constant $K \in \mathbf{R}_{0+}$;

(2) $T^{+}: \bigcup_{i \in \bar{p}} A_{i} \rightarrow \bigcup_{i \in \bar{p}} A_{i}$ is such that $T^{+} T^{-}\left(A_{i} \cup\right.$ $\left.A_{i+1}\right) \subseteq A_{i} \cup A_{i+1} ; \forall i \in \bar{p}$ satisfies the constraint $d\left(T^{+}\left(T^{-} x\right), T^{+}\left(T^{-} y\right)\right) \leq m\left(T^{-} x, T^{-} y\right) d\left(T^{-} x, T^{-} y\right)$ for some given bounded function $m:\left(\bigcup_{i \in \bar{p}} A_{i}\right) \times$ $\left(\bigcup_{i \in \bar{p}} A_{i}\right) \rightarrow \mathbf{R}_{0+}$.

Note that $p$-semicyclic impulsive self-mappings satisfy the subsequent combined constraint as follows:

$$
\begin{gathered}
d(T x, T y) \leq m\left(T^{-} x, T^{-} y\right)[K d(x, y)+(1-K) D] \\
\forall x \in A_{i}, \quad \forall y \in A_{i+1}, \quad \forall i \in \bar{p} ;
\end{gathered}
$$

then $T: \bigcup_{i \in \bar{p}} A_{i} \rightarrow \bigcup_{i \in \bar{p}} A_{i}$ which follows after combining the two ones given in Definition 1.

The following specializations of the $p$-semicyclic impulsive self-mapping $T: \bigcup_{i \in \bar{p}} A_{i} \rightarrow \bigcup_{i \in \bar{p}} A_{i}$ of Definition 1 are of interest.

(a) It is said to be nonexpansive (resp., contractive) $p$ semicyclic impulsive if, in addition, $K \in[0,1]$ (resp., if $K \in[0,1)$ ) and $m\left(T^{-} x, T^{-} y\right) \leq 1$.

(b) It is said to be $p$-cyclic impulsive if $T A_{i} \subseteq A_{i+1}, \forall i \in$ $\bar{p}$. It is said to be a nonexpansive (resp., contractive) $p$-cyclic impulsive if, in addition, $K \in[0,1]$ (resp., if $K \in[0,1))$ and $m\left(T^{-} x, T^{-} y\right) \leq 1$.

(c) It is said to be strictly p-semicyclic impulsive selfmapping if it satisfies the more stringent constraint

$$
\begin{aligned}
& d(T x, T y) \leq K m\left(T^{-} x, T^{-} y\right) d(x, y) \\
&+\left(1-K m\left(T^{-} x, T^{-} y\right)\right) D, \\
& \forall x \in A_{i}, \quad \forall y \in A_{i+1}, \quad \forall i \in \bar{p} .
\end{aligned}
$$

A motivation for such a concept is direct since $T$ : $\bigcup_{i \in \bar{p}} A_{i} \rightarrow \bigcup_{i \in \bar{p}} A_{i}$ is nonexpansive (resp., contractive) if $K m\left(T^{-} x, T^{-} y\right) \leq 1$ (resp., if $K m\left(T^{-} x, T^{-} y\right)<1$ ), $\forall x \in A_{i}$, $\forall y \in A_{i+1}$, and $\forall i \in \bar{p}$. This motivates, as a result, the concepts of nonexpansive and contractive strictly p-semicyclic impulsive self-mappings and the parallel ones of nonexpansive and contractive strictly $p$-cyclic impulsive self-mappings for the particular case that $A_{i} \subseteq A_{i+1}, \forall i \in \bar{p}$.

Remark 2. Note that if $m\left(T^{-} x, T^{-} y\right) \leq 1, \forall x \in A_{i}, \forall y \in$ $A_{i+1}$, and $\forall i \in \bar{p}$, then $m\left(T^{-} x, T^{-} y\right)(1-K) D \leq(1-$ $\left.K m\left(T^{-} x, T^{-} y\right)\right) D, \forall x \in A_{i}, \forall y \in A_{i+1}$, and $\forall i \in \bar{p}$, and this holds if $D=0$ (i.e., $\bigcap_{i \in \bar{p}} A_{i} \neq \emptyset$ ) irrespective of the value of $m\left(T^{-} x, T^{-} y\right), \forall x \in A_{i}, \forall y \in A_{i+1}$, and $\forall i \in \bar{p}$.

The subsequent result follows directly from Remark 2 .

Proposition 3. Assume that any of the two conditions below holds:

(1) $\bigcap_{i \in \bar{p}} A_{i} \neq \emptyset$;

(2) $\bigcap_{i \in \bar{p}} A_{i}=\emptyset$ and $0 \leq m\left(T^{-} x, T^{-} y\right) \leq 1, \forall x \in A_{i}$, $\forall y \in A_{i+1}$, and $\forall i \in \bar{p}$. 
Then, the self-mapping $T: \bigcup_{i \in \bar{p}} A_{i} \rightarrow \bigcup_{i \in \bar{p}} A_{i}$ is

(i) strictly p-semicyclic if it is p-semicyclic;

(ii) strictly nonexpansive (resp., contractive) p-semicyclic if it is nonexpansive (resp., contractive) p-semicyclic;

(iii) strictly $p$-cyclic if it is $p$-cyclic;

(iv) strictly nonexpansive (resp., contractive) p-cyclic if it is nonexpansive (resp., contractive) p-cyclic.

It is of interest the study of weaker properties than the above ones in an asymptotic context to be then able to investigate the asymptotic properties of distances for sequences $\left\{x_{n}\right\}_{n \in \mathbf{Z}_{0+}}$ of iterates built through $T: \bigcup_{i \in \bar{p}} A_{i} \rightarrow$ $\bigcup_{i \in \bar{p}} A_{i}$ according to $x_{n+1}=T x_{n}$ for all $n \in \mathbf{Z}_{0+}$ and some $x_{0} \in \bigcup_{i \in \bar{p}} A_{i}$ as well as the existence and uniqueness of fixed and best proximity points.

Lemma 4. Consider the p-semicyclic impulsive self-mapping $T: \bigcup_{i \in \bar{p}} A_{i} \rightarrow \bigcup_{i \in \bar{p}} A_{i}$ with $K \in[0,1]$, and define

$$
\begin{aligned}
& m^{\prime}\left(T^{-} x, T^{-} y\right)=m\left(T^{-} x, T^{-} y\right)-1, \\
& \delta_{k}(x)= m^{\prime}\left(T^{(k+1)-} x, T^{k-} x\right) \\
& \times\left(K d\left(T^{k} x, T^{k-1} x\right)+(1-K) D\right),
\end{aligned}
$$

for $x$ and $y$ in adjacent subsets $A_{i}$ and $A_{i+1}$ of $X$ for any $i \in \bar{p}$. Then, the following properties hold.

(i) The sequence $\left\{d\left(T^{k+n p+j} x, T^{k+n p+j-1} x\right)\right\}_{k \in \mathbf{Z}_{0+}}$ is bounded for all $k \in \mathbf{Z}_{0+}$, and $\forall n \in \mathbf{Z}_{+}, \forall j \in \overline{p-1} \cup\{0\}$ if

$$
\begin{aligned}
-d\left(T^{k+1} x, T^{k} x\right) \leq & \sum_{i \in S_{+}(k, n, j)} \delta_{k+j+n p-i}(x) \\
& -\sum_{i \in S_{-}(k, n, j)} \delta_{k+j+n p-i}(x)<\infty
\end{aligned}
$$

$\forall k \in \mathbf{Z}_{0+}, \quad \forall n \in \mathbf{Z}_{+}, \quad \forall j \in \overline{p-1} \cup\{0\}$,

where

$$
\begin{aligned}
& \delta_{k}(x)= m^{\prime}\left(T^{(k+1)-} x, T^{k-} x\right) \\
& \times\left(K d\left(T^{k} x, T^{k-1} x\right)+(1-K) D\right), \\
& \forall k \in \mathbf{Z}_{0+},
\end{aligned}
$$

Proof. Build a sequence of iterates $\left\{T^{k} x\right\}_{k \in \mathbf{Z}}$ according to $T T^{k-1} x=T^{+} T^{-} T^{k-1} x$ with $T^{0-} x=x, T^{0} x=T^{0+} T^{0-} x=x$, for any given $x \in A_{i}$ and any $i \in \bar{p}$ that is, $T=T^{0+}=T^{0-}=i d$ so that

$$
\begin{aligned}
d\left(T^{k+1} x, T^{k} x\right) \leq & \left(1+m^{\prime}\left(T^{(k+1)-} x, T^{k-} x\right)\right) \\
& \times d\left(T^{(k+1)-} x, T^{k-} x\right)
\end{aligned}
$$




$$
\begin{aligned}
& \leq\left(1+m^{\prime}\left(T^{(k+1)-} x, T^{k-} x\right)\right) \\
& \times\left(K d\left(T^{k} x, T^{k-1} x\right)+(1-K) D\right) \\
&= K d\left(T^{k} x, T^{k-1} x\right)+(1-K) D+\delta_{k}(x), \\
& \forall k \in \mathbf{Z}_{0+} .
\end{aligned}
$$

Through a recursive calculation with (4), one get:

$$
\begin{aligned}
0 \leq & d\left(T^{k+n p+j} x, T^{k+n p+j-1} x\right) \\
\leq & K d\left(T^{k+n p+j-1} x, T^{k+n p+j-2} x\right) \\
& +(1-K) D+\delta_{k+n p+j-1}(x) \\
\leq & K^{2} d\left(T^{k+n p+j-2} x, T^{k+n p+j-3} x\right) \\
& +K\left[(1-K) D+\delta_{k+n p+j-2}(x)\right] \\
& +(1-K) D+\delta_{k+n p+j-1}(x) \\
\leq & \cdots \leq K^{n p+j-1} d\left(T^{k+1} x, T^{k} x\right) \\
& +\left(1-K^{n p+j-1}\right) D+\sum_{i=1}^{n p+j} K^{i} \delta_{k+n p+j-i}(x)
\end{aligned}
$$

$\forall k \in \mathbf{Z}_{0+}, \quad \forall n \in \mathbf{Z}_{+}, \quad \forall j \in \overline{p-1} \cup\{0\}$.

If $K=1$, then

$$
\begin{aligned}
0 \leq & d\left(T^{k+n p+j} x, T^{k+n p+j-1} x\right) \leq d\left(T^{k+1} x, T^{k} x\right) \\
& +\sum_{i \in S_{+}(k, n, j)} \delta_{k+n p+j-i}(x)-\sum_{i \in S_{-}(k, n, j)} \delta_{k+n p+j-i}(x),
\end{aligned}
$$

$\forall k \in \mathbf{Z}_{0+}, \quad \forall n \in \mathbf{Z}_{+}, \quad \forall j \in \overline{p-1} \cup\{0\}$.

Take any $k \in \mathbf{Z}_{0+}$, any $n \in \mathbf{Z}_{+}$, and any $x \in \bigcup_{i \in \bar{p}} A_{i}$. Since $d\left(T^{k+1} x, T^{k} x\right)$ is finite and (4) holds, it follows that $0 \leq d\left(T^{k+n p+j+1} x, T^{k+n p+j} x\right)<\infty$. If, in addition, $T:$ $\bigcup_{i \in \bar{p}} A_{i} \rightarrow \bigcup_{i \in \bar{p}} A_{i}$ is $p$-cyclic, then the zero lower-bound of (7) is replaced with $D$. If $T: \bigcup_{i \in \bar{p}} A_{i} \rightarrow \bigcup_{i \in \bar{p}} A_{i}$ is $p$-semicyclic (in particular, $p$-cyclic) nonexpansive, then (4) always holds since $m\left(T^{(k+n p+j+i)-} x, T^{(k+n p+j+i-1)-} x\right) \leq 1,-1 \leq$ $m^{\prime}\left(T^{(k+j+n p-i+1)-} x, T^{(k+j+n p-i)-} x\right) \leq 0$ so that

$$
\begin{gathered}
\sum_{i \in S_{+}(k, n, j)} \delta_{k+j+n p-i}(x)-\sum_{i \in S_{-}(k, n, j)} \delta_{k+j+n p-i}(x) \\
=\sum_{i \in S_{-}(k, n, j)} \delta_{k+j+n p-i}(x) \leq 0,
\end{gathered}
$$

if $m\left(T^{(k+j+n p-i+1)-} x, T^{(k+j+n p-i)-} x\right)=1$ and $\left\{d\left(T^{k+n p+j+1} x\right.\right.$, $\left.\left.T^{k+n p+j} x\right)\right\}_{k \in \mathbf{Z}_{0+}}$ is always bounded; $\forall k \in \mathbf{Z}_{0+}, \forall n \in \mathbf{Z}_{+}$, and $\forall j \in \overline{p-1} \cup\{0\}$. Property (i) has been proven. If $K \in[0,1)$, then

$$
\begin{aligned}
0 \leq & d\left(T^{k+n p+j} x, T^{k+n p+j-1} x\right) \\
\leq & K^{n+j-1} d\left(T^{k+1} x, T^{k} x\right)+\left(1-K^{n p+j-1}\right) D \\
& +\sum_{i \in S_{+}(k, n, j)} \delta_{k+j+n p-i}(x)-\sum_{i \in S_{-}(k, n, j)} \delta_{k+j+n p-i}(x) \\
0 \leq & \limsup _{n \rightarrow \infty} d\left(T^{k+n p+j} x, T^{k+n p+j-1} x\right) \\
\leq & D+\limsup _{n \rightarrow \infty} \sum_{i=1}^{n p+j} \delta_{k+j+n p-i}(x) .
\end{aligned}
$$

If, in addition, $T: \bigcup_{i \in \bar{p}} A_{i} \rightarrow \bigcup_{i \in \bar{p}} A_{i}$ is $p$-cyclic, then the zero lower-bound of (13)-(14) is replaced with $D$.

If $T: \bigcup_{i \in \bar{p}} A_{i} \rightarrow \bigcup_{i \in \bar{p}} A_{i}$ is contractive $p$-semicyclic, then (14) becomes $0 \leq \lim \sup _{n \rightarrow \infty} d\left(T^{k+n p+1} x, T^{k+n p} x\right) \leq$ $D$ from (12). If, in addition, $T: \bigcup_{i \in \bar{p}} A_{i} \rightarrow \bigcup_{i \in \bar{p}} A_{i}$ is contractive $p$-cyclic, then $D \leq \lim \sup _{n \rightarrow \infty} d\left(T^{k+n p+1} x\right.$, $\left.T^{k+n p} x\right) \leq D, \forall x \in \bigcup_{i \in \bar{p}} A_{i}$ so that there is $\lim _{n \rightarrow \infty}$ $d\left(T^{k+n p+1} x, T^{k+n p} x\right)=D, \forall x \in \bigcup_{i \in \bar{p}} A_{i}$. Property (ii) has been proven.

The following result establishes an asymptotic property of the limits superiors of distances of consecutive points of the iterated sequences which implies that $T: \bigcup_{i \in \bar{p}} A_{i} \rightarrow$ $\bigcup_{i \in \bar{p}} A_{i}$ is asymptotically contractive, and the limit $\lim _{n \rightarrow \infty}\left(\sum_{k=0}^{n p+j-2}\left(\prod_{\ell=k}^{n p+j-2}\left[K_{\ell+i}\right]\right)\left(m\left(T^{(k+1)-} x, T^{k-} x\right)-1\right)\right)=$ $0, \forall x \in \bigcup_{i \in \bar{p}} A_{i}, \forall j \in \overline{p-1} \cup\{0\}$ exists. In particular, it is not required that $m(x, y) \leq 1$ for any $x \in A_{i}, y \in A_{i+1}$, and $\forall i \in$ $\bar{p}$ as in contractive and, in general, nonexpansive $p$-semicyclic impulsive self-mappings.

Theorem 5. Consider the following generalization of condition 3 of Definition 1:

$$
D \leq d\left(T^{2-} x, T^{-} y\right) \leq K_{i} d(T x, x)+\left(1-K_{i}\right) D
$$

for any given $x \in A_{i}, \forall i \in \bar{p}$, and define $\bar{K}=\prod_{i=1}^{p-1}\left[K_{i}\right]$. Define

$\widehat{K}$

$$
\begin{aligned}
& =\bar{K} \sup _{x \in \bigcup_{i \in \bar{p}}} \max _{A_{i}}\left(\prod_{n \in \mathbf{Z}_{0+}}^{(n+1) p-1}\left[m\left(T^{(i+1)-} x, T^{i-} x\right)\right]\right) \\
& =\prod_{i=1}^{p-1}\left[K_{i}\right] \sup _{x \in \bigcup_{k \in \bar{p}}} \max _{k}\left(\prod_{n \in \mathbf{Z}_{0+}}^{(n+1) p-1}\left[m \times\left(T^{(i+1)-} x, T^{i-} x\right)\right]\right),
\end{aligned}
$$

such that $\widehat{K} \in[0,1)$. Then, the following properties hold. 
(i)

$$
\begin{aligned}
& D_{0} \leq \limsup _{n \rightarrow \infty} d\left(T^{n p+j} x, T^{n p+j-1} x\right) \\
& \leq\left(1+\frac{1}{1-\widehat{K}}\left(\prod_{\ell=0}^{j-1}\left[K_{i+\ell}\right]\right)\right. \\
&\left.\quad \times \sup _{x \in \bigcup_{k \in \bar{p}} A_{k}} \max _{\ell \in \mathbf{Z}_{0+}}\left|m^{\prime}\left(T^{(\ell+1)-} x, T^{\ell-} x\right)\right|\right) D, \\
& \forall x \in \bigcup_{i \in \bar{p}} A_{i}, \quad \forall j \in \overline{p-1} \cup\{0\},
\end{aligned}
$$$$
D_{0} \leq d\left(T^{n p+j} x, T^{n p+j-1} x\right)
$$$$
\leq\left(\prod_{\ell=0}^{j-1}\left[K_{i+\ell}\right]\right) \widehat{K}^{n} d(T x, x)
$$$$
+\left[\left(1-\left(\prod_{\ell=0}^{j-1}\left[K_{i+\ell}\right]\right) \widehat{K}^{n}\right)\right.
$$$$
+\frac{1-\widehat{K}^{n}}{1-\widehat{K}}\left(\prod_{\ell=0}^{j-1}\left[K_{i+\ell}\right]\right)
$$$$
\left.\times \sup _{x \in \bigcup_{k \in \bar{p}} A_{k}} \max _{\ell \in \mathbf{Z}_{0+}}\left|m^{\prime}\left(T^{(\ell+1)-} x, T^{\ell-} x\right)\right|\right] D,
$$

$\forall x \in A_{i}, \quad \forall i \in \bar{p}, \quad \forall j \in \overline{p-1} \cup\{0\}$,

where $D_{0}=0$ if $T: \bigcup_{i \in \bar{p}} A_{i} \rightarrow \bigcup_{i \in \bar{p}} A_{i}$ is p-semicyclic and $D_{0}=D$ if $T: \bigcup_{i \in \bar{p}} A_{i} \rightarrow \bigcup_{i \in \bar{p}} A_{i}$ is $p$-cyclic. that

(ii) If, furthermore, there is a real constant $\varepsilon_{0} \geq-1$ such

$$
\begin{aligned}
\limsup _{n \rightarrow \infty}\left(\sum_{k=0}^{n p+j-2}\left(\prod_{\ell=k-1}^{n p+j-2}\left[K_{\ell+i}\right]\right)\right. \\
\left.\times\left(m\left(T^{(k+1)-} x, T^{k-} x\right)-1\right)\right) \leq \varepsilon_{0}, \\
\forall x \in \bigcup_{i \in \bar{p}} A_{i}, \quad \forall j \in \overline{p-1} \cup\{0\},
\end{aligned}
$$

then

$$
\begin{gathered}
D_{0} \leq \limsup _{n \rightarrow \infty} d\left(T^{n p+j} x, T^{n p+j-1} x\right) \\
\leq D\left(1+\varepsilon_{0}\right), \quad \forall x \in \bigcup_{i \in \bar{p}} A_{i}, \\
\forall j \in \overline{p-1} \cup\{0\} .
\end{gathered}
$$

$$
\begin{aligned}
& +\left(1-\left(\prod_{i=1}^{p-1}\left[m\left(T^{(i+1)-} x, T^{i-} x\right)\right]\right) \bar{K}\right) D \\
& +\left(\sum_{k=0}^{p-2}\left(\prod_{\ell=k-1}^{p-2}\left[K_{\ell+i}\right]\right) m^{\prime}\left(T^{(k+1)-} x, T^{k-} x\right)\right) D \\
d\left(T^{n p+j} x, T^{n p+j-1} x\right) & \left(\prod_{i=1}^{n p+j-1}\left[m\left(T^{(i+1)-} x, T^{i-} x\right)\right]\right)\left(\prod_{\ell=0}^{j-1}\left[K_{i+\ell}\right]\right) \\
& \times \bar{K}^{n} d(T x, x) \\
& +\left(1-\left(\prod_{i=1}^{n p+j-1}\left[m\left(T^{(i+1)-} x, T^{i-} x\right)\right]\right)\right. \\
& \left.\times\left(\prod_{\ell=0}^{j-1}\left[K_{i+\ell}\right]\right) \bar{K}^{n}\right) D \\
& +\left(\sum_{k=0}^{n p+j-2}\left(\prod_{\ell=k-1}^{n p+j-2}\left[K_{\ell+i}\right]\right) m^{\prime}\left(T^{(k+1)-} x, T^{k-} x\right)\right) D,
\end{aligned}
$$$$
\forall x \in A_{i}, \quad \forall i \in \bar{p}, \quad \forall j \in \overline{p-1} \cup\{0\},
$$

Proof. Since $\widehat{K} \in[0,1)$, one has through iterative calculation via (15)

$$
\begin{aligned}
d\left(T^{2} x, T x\right) \leq & m\left(T^{2-} x, T^{-} x\right)\left(K_{i} d(T x, x)+(1-K) D\right) \\
= & \left(m\left(T^{2-} x, T^{-} x\right) K_{i}\right) d(T x, x) \\
& +\left(1-m\left(T^{2-} x, T^{-} x\right) K_{i}\right) D \\
& +m^{\prime}\left(T^{2-} x, T^{-} x\right) D,
\end{aligned}
$$$$
d\left(T^{p} x, T^{p-1} x\right)
$$$$
\leq\left(\prod_{i=1}^{p-1}\left[m\left(T^{(i+1)-} x, T^{i-} x\right)\right]\right) \bar{K} d(T x, x)
$$

$\forall x \in A_{i}, \quad \forall i \in \bar{p}, \quad \forall j \in \overline{p-1} \cup\{0\}$,

with the convention $\left(\prod_{\ell=0}^{-1}\left[K_{i+\ell}\right]\right)=1, \forall i \in \bar{p}$. Then, one gets (17), and Property (i) has been proven. To prove Property (ii), use the indicator sets (6) and, since $m^{\prime}\left(T^{2-} x, T^{-} x\right) \geq-1$, $\forall x \in \bigcup_{i \in \bar{p}} A_{i}$, one also gets from (15)-(16)

$$
\begin{array}{r}
D_{0}+\left[\operatorname { l i m i n f } _ { n \rightarrow \infty } \left(\sum_{k \in S_{-}(k, n, j-2)}\left(\prod_{\ell=k}^{n p+j-2}\left[K_{\ell+i}\right]\right)\right.\right. \\
\left.\left.\times\left|m^{\prime}\left(T^{(k+1)-} x, T^{k-} x\right)\right|\right)\right] D
\end{array}
$$




$$
\begin{aligned}
& \leq \limsup _{n \rightarrow \infty} d\left(T^{n p+j} x, T^{n p+j-1} x\right) \\
& +\left[\operatorname { l i m i n f } _ { n \rightarrow \infty } \left(\sum_{k \in S_{-}(k, n, j-2)}\left(\prod_{\ell=k-1}^{n p+j-2}\left[K_{\ell+i}\right]\right)\right.\right. \\
& \left.\left.\times\left|m^{\prime}\left(T^{(k+1)-} x, T^{k-} x\right)\right|\right)\right] D \\
& \leq D\left[\operatorname { l i m s u p } _ { n \rightarrow \infty } \left(1+\left(\sum_{k \in S_{+}(k, n, j-2)}\left(\prod_{\ell=k-1}^{n p+j-2}\left[K_{\ell+i}\right]\right)\right.\right.\right. \\
& \left.\times m^{\prime}\left(T^{(k+1)-} x, T^{k-} x\right)\right) \\
& \forall x \in \bigcup_{i \in \bar{p}} A_{i}, \forall j \in \overline{p-1} \cup\{0\},
\end{aligned}
$$

and (19), and then Property (ii), follows from (18).

Note from (19) in Theorem 5 that if $D_{0}=D=0$, that is, $\bigcap_{i \in \bar{p}} A_{i} \neq \emptyset$, and $\varepsilon_{0} \in[-1, \infty)$, then $\exists \lim _{n \rightarrow \infty} d\left(T^{n p+j} x\right.$, $\left.T^{n p+j-1} x\right)=0, \forall x \in \bigcup_{i \in \bar{p}} A_{i}, \forall j \in \overline{p-1} \cup\{0\}$ from (19) since $\widehat{K} \in[0,1)$. In this case, $T: \bigcup_{i \in \bar{p}} A_{i} \rightarrow \bigcup_{i \in \bar{p}} A_{i}$ is an asymptotically contractive $p$-cyclic (and also $p$-semicyclic since $D=0$ ) self-mapping on the union on intersecting closed subsets of $X$. A close property follows if $D_{0}=D \neq 0$, and $\varepsilon_{0}=0$ implying from (19) that

$$
\begin{gathered}
\limsup _{n \rightarrow \infty}\left(\sum_{k=0}^{n p+j-2}\left(\prod_{\ell=k-1}^{n p+j-2}\left[K_{\ell+i}\right]\right)\right. \\
\left.\quad \times\left(m\left(T^{(k+1)-} x, T^{k-} x\right)-1\right)\right) \\
=\lim _{n \rightarrow \infty}\left(\sum_{k=0}^{n p+j-2}\left(\prod_{\ell=k-1}^{n p+j-2}\left[K_{\ell+i}\right]\right)\right. \\
\left.\quad \times\left(m\left(T^{(k+1)-} x, T^{k-} x\right)-1\right)\right)=0, \\
\forall x \in \bigcup_{i \in \bar{p}} A_{i}, \quad \forall j \in \overline{p-1} \cup\{0\}
\end{gathered}
$$

and leading to $\exists \lim _{n \rightarrow \infty} d\left(T^{n p+j} x, T^{n p+j-1} x\right)=D$ such that $T$ : $\bigcup_{i \in \bar{p}} A_{i} \rightarrow \bigcup_{i \in \bar{p}}^{n} A_{i}$ is a contractive $p$-cyclic self-mapping on the union on disjoint closed subsets of $X$. The above discussion is summarized in the subsequent result.
Corollary 6. Assume that (15) holds with $\widehat{K}$ defined in (16) being in $[0,1)$, and assume also that

$$
\begin{array}{r}
\infty>\varepsilon_{0} \\
\geq \max \left(\operatorname { l i m s u p } _ { n \rightarrow \infty } \left(\sum_{k=0}^{n p+j-2}\left(\prod_{\ell=k-1}^{n p+j-2}\left[K_{\ell+i}\right]\right)\right.\right. \\
\left.\left.\times\left(m\left(T^{(k+1)-} x, T^{k-} x\right)-1\right),-1\right)\right), \\
\forall x \in \bigcup_{i \in \bar{p}} A_{i}, \quad \forall j \in \overline{p-1} \cup\{0\} .
\end{array}
$$

Then, the following properties hold

(i) If $\bigcap_{i \in \bar{p}} A_{i} \neq \emptyset$, then $T: \bigcup_{i \in \bar{p}} A_{i} \rightarrow \bigcup_{i \in \bar{p}} A_{i}$ is an asymptotically contractive $p$-cyclic impulsive self-mapping so that there is the limit

$$
\begin{aligned}
& \lim _{n \rightarrow \infty} d\left(T^{n p+j} x, T^{n p+j-1} x\right)=0, \\
& \forall x \in \bigcup_{i \in \bar{p}} A_{i}, \quad \forall j \in \overline{p-1} \cup\{0\} .
\end{aligned}
$$

(ii) If $\bigcap_{i \in \bar{p}} A_{i}=\emptyset, d(T x, T y) \geq D, \forall x \in A_{i}, \forall y \in A_{i+1}$, and $\forall i \in \bar{p}$ and the following limit exists:

$$
\begin{aligned}
\lim _{n \rightarrow \infty}\left(\sum_{k=0}^{n p+j-2}\left(\prod_{\ell=k-1}^{n p+j-2}\left[K_{\ell+i}\right]\right)\right. \\
\left.\times\left(m\left(T^{(k+1)-} x, T^{k-} x\right)-1\right)\right)=0 ; \\
\forall x \in \bigcup_{i \in \bar{p}} A_{i}, \quad \forall j \in \overline{p-1} \cup\{0\}
\end{aligned}
$$

then $T: \bigcup_{i \in \bar{p}} A_{i} \rightarrow \bigcup_{i \in \bar{p}} A_{i}$ is an asymptotically contractive p-cyclic impulsive self-mapping so that the limit

$$
\begin{array}{r}
\lim _{n \rightarrow \infty} d\left(T^{n p+j} x, T^{n p+j-1} x\right)=D, \\
\forall x \in \bigcup_{i \in \bar{p}} A_{i}, \quad \forall j \in \overline{p-1} \cup\{0\} \text { exists. }
\end{array}
$$

A particular result got from Theorem 5 follows for contractive $p$-semicyclic and $p$-cyclic impulsive self-mappings $T: \bigcup_{i \in \bar{p}} A_{i} \rightarrow \bigcup_{i \in \bar{p}} A_{i}$.

Corollary 7. Theorem 5 holds with $D_{0}=0$ if $T: \bigcup_{i \in \bar{p}} A_{i} \rightarrow$ $\bigcup_{i \in \bar{p}} A_{i}$ is contractive $p$-semicyclic and with $D_{0}=D$ if the impulsive self-mapping $T: \bigcup_{i \in \bar{p}} A_{i} \rightarrow \bigcup_{i \in \bar{p}} A_{i}$ is contractive p-cyclic provided that $\bar{K}=\prod_{i=1}^{p-1}\left[K_{i}\right] \in[0,1)$.

Proof. It is a direct consequence of Theorem 5 since $\bar{K}=$ $\prod_{i=1}^{p-1}\left[K_{i}\right] \in[0,1)$ implies that $\widehat{K} \in[0,1)$ since $m\left(T^{-} x\right.$, $\left.T^{-} y\right) \leq 1, \forall x \in A_{i}, \forall y \in A_{i+1}$, and $\forall i \in \bar{p}$. 
Remark 8. Note that if $T: \bigcup_{i \in \bar{p}} A_{i} \rightarrow \bigcup_{i \in \bar{p}} A_{i}$ is a nonexpansive $p$-cyclic impulsive self-mapping, the following constraints hold:

$$
\begin{gathered}
m\left(T x^{-}, T y^{-}\right) \leq 1, \\
D \leq m\left(T x^{-}, T y^{-}\right)(K d(x, y)-D)+m\left(T x^{-}, T y^{-}\right) D, \\
\forall x \in A_{i}, \quad \forall y \in A_{i+1}, \quad \forall i \in \bar{p},
\end{gathered}
$$

and equivalently,

$$
\begin{aligned}
1 & \geq m\left(T x^{-}, T y^{-}\right) \\
& \geq \frac{D}{K d(x, y)+(1-K) D} \\
& =\frac{D}{D+K(d(x, y)-D)},
\end{aligned}
$$

implying that

(a) $1 \geq m\left(T x^{-}, T y^{-}\right) \geq 0, \forall x \in A_{i}, \forall y \in A_{i+1}$, and $\forall i \in \bar{p}$ if $D=0$; that is, if the sets $A_{i}$ intersect $\forall i \in \bar{p}$.

(b) $m\left(T x^{-}, T y^{-}\right)=1$ if $d(x, y)=D$; that is, for best proximity points associated with any two adjacent disjoint subsets $A_{i}, y \in A_{i+1}$ for $i \in \bar{p}$.

On the other hand, note that Corollary 6 (ii) implies the asymptotic convergence of distances in-between consecutive points of the iterated sequences generated via $T: \bigcup_{i \in \bar{p}} A_{i} \rightarrow$ $\bigcup_{i \in \bar{p}} A_{i}$ to the distance $D$ between adjacent sets. This property does not imply $1 \geq m\left(T x^{-}, T y^{-}\right), \forall x \in A_{i}$, and $\forall y \in A_{i+1}$, $\forall i \in \bar{p}$ as required for nonexpansive (and, in particular, for contractive) $p$-cyclic impulsive self-mappings. However, it implies $m\left(T^{(n+1)-} x, T^{n-} x\right) \rightarrow 1$ as $n \rightarrow \infty$ from (25), since the sequence defining its left-hand-side sequence has to converge asymptotically to zero.

Define recursively global functions to evaluate the nonexpansive and contractive properties of the impulsive selfmapping $T: \bigcup_{i \in \bar{p}} A_{i} \rightarrow \bigcup_{i \in \bar{p}} A_{i}$ which take into account the most general case that the constant $K$ in Definition 1 (1) can be generalized to be set dependent and point-dependent leading to a combined extended constraint as follows:

$$
\begin{array}{r}
d\left(T^{2} x, T x\right) \leq K_{i}(x, T x) m\left(T^{2-} x, T^{-} x\right) d(x, T x) \\
+m\left(T^{2-} x, T^{-} x\right)\left(1-K_{i}(x, T x)\right) D, \\
\forall x \in \bigcup_{i \in \bar{p}} A_{i},
\end{array}
$$

so that

$$
\begin{aligned}
& \widehat{K}^{(j)}(x, T x) \\
&=\left(\prod _ { i = 1 } ^ { p - 1 } \left[m\left(T^{(i+j p+1)-} x, T^{(i+j p)-} x\right)\right.\right. \\
&\left.\left.\quad \times K_{i}\left(T^{i+j p} x, T^{i+j p-1} x\right)\right]\right) \widehat{K}^{(j-1)}(x)
\end{aligned}
$$

$$
\forall x \in \bigcup_{i \in \bar{p}} A_{i}, \quad \forall j \in \mathbf{Z}_{+},
$$

with $x=T^{0} x$ and initial, in general, point-dependent value

$$
\begin{aligned}
& \widehat{K}^{(0)}(x, T x)=\prod_{i=1}^{p-1}[ m\left(T^{(i+1)-} x, T^{(i)-} x\right) \\
&\left.\times K_{i}\left(T^{i} x, T^{i-1} x\right)\right], \\
& \forall x \in \bigcup_{i \in \bar{p}} A_{i}, \quad \forall j \in \mathbf{Z}_{+},
\end{aligned}
$$

for each iterated sequence constructed through the impulsive self-mapping $T: \bigcup_{i \in \bar{p}} A_{i} \rightarrow \bigcup_{i \in \bar{p}} A_{i}$. The following related result follows.

Theorem 9. Consider the p-semicyclic impulsive self-mapping $T: \bigcup_{i \in \bar{p}} A_{i} \rightarrow \bigcup_{i \in \bar{p}} A_{i}$ under the constraint (29) subject to (30)-(31). If $\lim _{n \rightarrow \infty} \widehat{K}^{(n)}(x, T x)=0, \forall x \in \bigcup_{i \in \bar{p}} A_{i}$, then the following properties hold.

(i) If $\bigcap_{i \in \bar{p}} A_{i} \neq \emptyset$ then

$$
\begin{array}{r}
\lim _{n \rightarrow \infty} d\left(T^{(n+1) p} x, T^{(n+1) p-1} x\right)=0 ; \\
\forall x \in \bigcup_{i \in \bar{p}} A_{i}, \quad \forall j \in \overline{p-1} \cup\{0\}, \quad \forall n \in \mathbf{Z}_{0+}
\end{array}
$$

so that $T: \bigcup_{i \in \bar{p}} A_{i} \rightarrow \bigcup_{i \in \bar{p}} A_{i}$ is asymptotically contractive $p$-semicyclic cyclic in the sense that, given $x \in A_{i}$, there is a sufficiently large $n_{0}=n_{0}(x) \in \mathbf{Z}_{0+}$ such that, together with (32), $T^{n p} x \in A_{i}, T^{(n+1) p} x \in A_{i} \cup A_{i+1}$ for $n \geq n_{0}$.

(ii) If $\bigcap_{i \in \bar{p}} A_{i}=\emptyset$ and the limit below exists:

$$
\begin{aligned}
& \lim _{n \rightarrow \infty} \sum_{j=0}^{n-1} \widehat{K}^{(n-j)}(x, T x) \\
& \times\left(\sum_{k=j p}^{(j+1) p-2}\left(\prod_{\ell=k+i-1}^{(j+1) p-2}\left[K_{\ell}\left(T^{(k-1)} x, T^{k} x\right)\right]\right)\right. \\
&\left.\times\left(m\left(T^{(k+1)-} x, T^{k-} x\right)-1\right)\right)=0, \\
& \forall x \in \bigcup_{i \in \bar{p}} A_{i},
\end{aligned}
$$


then

$$
\lim _{n \rightarrow \infty} d\left(T^{(n+1) p} x, T^{(n+1) p-1} x\right)=D, \quad \forall x \in \bigcup_{i \in \bar{p}} A_{i}
$$

$\forall j \in \overline{p-1} \cup\{0\}, \quad \forall n \in \mathbf{Z}_{0+}$, and $T: \bigcup_{i \in \bar{p}} A_{i} \rightarrow \bigcup_{i \in \bar{p}} A_{i}$ is asymptotically contractive $p$ cyclic in the sense that, given $x \in A_{i}$, together with (34), there is a sufficiently large $n_{0}=n_{0}(x) \in \mathbf{Z}_{0+}$ such that, together with (34), $T^{n p} x \in A_{i}, T^{(n+1) p} x \in A_{i+1}$ for $n \geq n_{0}$.

(iii) The limit (33) exists and then (34) holds if $m:\left(\bigcup_{i \in \bar{p}} A_{i}\right) \times\left(\bigcup_{i \in \bar{p}} A_{i}\right) \rightarrow \mathbf{R}_{0+}$ satisfies the identity

$$
\begin{aligned}
m\left(T^{((n+1) p-1)-} x,\right. & \left.T^{((n+1) p-2)-} x\right) \\
=1+\varepsilon_{n}- & \frac{1}{\widehat{K}^{(1)}(x, T x)\left(\sum_{k=n p}^{(n+1) p-2}\left(\prod_{\ell=(n+1) p+i-3}^{(n+1) p-2}\left[K_{\ell}\left(T^{((n+1) p-3)} x, T^{(n+1) p-2} x\right)\right]\right)\right)} \\
& \times\left(\widehat{K}^{(1)}(x, T x)\left(\sum_{k=n p}^{(n+1) p-3}\left(\prod_{\ell=k+i-1}^{(n+1) p-2}\left[K_{\ell}\left(T^{(k-1)} x, T^{k} x\right)\right]\right)\left(m\left(T^{(k+1)-} x, T^{k-} x\right)-1\right)\right)\right. \\
& \left.+\sum_{j=0}^{n-1} \widehat{K}^{(n-j)}(x, T x)\left(\sum_{k=j p}^{(j+1) p-2}\left(\prod_{\ell=k+i-1}^{(j+1) p-2}\left[K_{\ell}\left(T^{(k-1)} x, T^{k} x\right)\right]\right)\left(m\left(T^{(k+1)-} x, T^{k-} x\right)-1\right)\right)\right) .
\end{aligned}
$$

Proof. One gets from (20), (29)-(31) that

$$
\begin{aligned}
d( & \left.T^{p} x, T^{p-1} x\right) \\
\leq & \widehat{K}^{(0)}(x, T x) d(T x, x) \\
& +\left(1-\widehat{K}^{(0)}(x, T x)\right) D \\
& +\left(\sum_{k=0}^{p-2}\left(\prod_{\ell=k+i-1}^{p-2}\left[K_{\ell}\left(T^{(k-1)} x, T^{k} x\right)\right]\right)\right. \\
d\left(T^{(n+1) p} x, T^{(n+1) p-1} x\right) & \left.\times m^{\prime}\left(T^{(k+1)-} x, T^{k-} x\right)\right) D, \\
\leq & \widehat{K}^{(n)}(x, T x) d(T x, x)+\left(1-\widehat{K}^{(n)}(x, T x)\right) D \\
& +\sum_{j=0}^{n-1} \widehat{K}^{(n-j)}(x, T x) \\
& \times\left(\sum_{k=j p}^{(j+1) p-2}\left(\prod_{\ell=k+i-1}^{(j+1) p-2}\left[K_{\ell}\left(T^{(k-1)} x, T^{k} x\right)\right]\right)\right.
\end{aligned}
$$

$\forall x \in A_{i}, \quad \forall i \in \bar{p}, \quad \forall j \in \overline{p-1} \cup\{0\}, \quad \forall n \in \mathbf{Z}_{0+}$, where $m^{\prime}\left(T^{(n+1)-} x, T^{n-} x\right)=m\left(T^{(n+1)-} x, T^{n-} x\right)-1$. If $\lim _{n \rightarrow \infty} \widehat{K}^{(n)}(x, T x)=0, \forall x \in \bigcup_{i \in \bar{p}} A_{i}$ and (33) holds, then $d\left(T^{(n+1) p} x, T^{(n+1) p-1} x\right) \rightarrow D$ as $n \rightarrow \infty, \forall x \in \bigcup_{i \in \bar{p}} A_{i}$, $\forall j \in \overline{p-1} \cup\{0\}$, and $\forall n \in \mathbf{Z}_{0+}$. This leads directly to Property (i) for $D=0$ if $\bigcap_{i \in \bar{p}} A_{i} \neq \emptyset$ (without the constraint (33) being needed) and to Property (ii) for $D \neq 0$ if $\bigcap_{i \in \bar{p}} A_{i}=\emptyset$.

Consider that

$$
\sum_{j=0}^{n} \widehat{K}^{(n+1-j)}(x, T x)
$$

$$
\begin{gathered}
\times\left(\sum_{k=j p}^{(j+1) p-2}\left(\prod_{\ell=k+i-1}^{(j+1) p-2}\left[K_{\ell}\left(T^{(k-1)} x, T^{k} x\right)\right]\right)\right. \\
\left.\times\left(m\left(T^{(k+1)-} x, T^{k-} x\right)-1\right)\right)
\end{gathered}
$$$$
=\widehat{K}^{(1)}(x, T x)
$$$$
\begin{gathered}
\times \sum_{k=n p}^{(n+1) p-2}\left(\prod_{\ell=(n+1) p+i-3}^{(n+1) p-2}\left[K_{\ell}\left(T^{((n+1) p-3)} x, T^{(n+1) p-2} x\right)\right]\right) \\
\left.\times\left(m\left(T^{((n+1) p-1)-} x, T^{((n+1) p-2)-} x\right)-1\right)\right)
\end{gathered}
$$

$$
+\widehat{K}^{(1)}(x, T x)
$$$$
\times\left(\sum_{k=n p}^{(n+1) p^{-3}}\left(\prod_{\ell=k+i-1}^{(n+1) p^{-2}}\left[K_{\ell}\left(T^{(k-1)} x, T^{k} x\right)\right]\right)\right.
$$

$$
\left.\times\left(m\left(T^{(k+1)-} x, T^{k-} x\right)-1\right)\right)
$$




$$
\begin{aligned}
& +\sum_{j=0}^{n-1} \widehat{K}^{(n-j)}(x, T x) \\
& \times\left(\sum_{k=j p}^{(j+1) p-2}\left(\prod_{\ell=k+i-1}^{(j+1) p-2}\left[K_{\ell}\left(T^{(k-1)} x, T^{k} x\right)\right]\right)\right. \\
& \left.\quad \times\left(m\left(T^{(k+1)-} x, T^{k-} x\right)-1\right)\right)
\end{aligned}
$$

converges to zero as $n \rightarrow \infty$ if for some real sequence $\left\{\varepsilon_{n}\right\}_{n \in \mathbf{Z}_{0+}}$ which converges to zero, the function $m:\left(\bigcup_{i \in \bar{p}}\right.$ $\left.A_{i}\right) \times\left(\bigcup_{i \in \bar{p}} A_{i}\right) \rightarrow \mathbf{R}_{0+}$ satisfies (35). This proves Property (iii).

Theorem 9 has a counterpart in terms of asymptotically strict $p$-semicyclic and cyclic versions established as follows.

Corollary 10. Assume that the following strict-type contractive condition holds:

$$
\begin{array}{r}
d\left(T^{2} x, T x\right) \leq K_{i}(x, T x) m\left(T^{2-} x, T^{-} x\right) d(x, T x) \\
+\left(1-m\left(T^{2-} x, T^{-} x\right) K_{i}(x, T x)\right) D \\
\forall x \in \bigcup_{i \in \bar{p}} A_{i}
\end{array}
$$

subject to the constraints (30) and (31). If $\lim _{n \rightarrow \infty} \widehat{K}^{(n)}$ $(x, T x)=0, \forall x \in \bigcup_{i \in \bar{p}} A_{i}$, then (34) holds, and $T:$ $\bigcup_{i \in \bar{p}} A_{i} \rightarrow \bigcup_{i \in \bar{p}} A_{i}$ is a strictly asymptotically contractive $p$ cyclic impulsive self-mapping in the sense that, given any $x \in$ $A_{i}$, there is a sufficiently large $n_{0}=n_{0}(x) \in \mathbf{Z}_{0+}$ such that, together with (34), $T^{n p} x \in A_{i}, T^{(n+1) p} x \in A_{i+1}$ for all $n \geq n_{0}$ if $\bigcap_{i \in \bar{p}} A_{i}=\emptyset$.

If $\bigcap_{i \in \bar{p}} A_{i} \neq \emptyset$, then $T: \bigcup_{i \in \bar{p}} A_{i} \rightarrow \bigcup_{i \in \bar{p}} A_{i}$ is (at least) strictly asymptotically contractive p-semicyclic in the sense that there is a sufficiently large $n_{0}=n_{0}(x) \in \mathbf{Z}_{0+}$ such that, together with (32), $T^{n p} x \in A_{i}, T^{(n+1) p} x \in A_{i} \cup A_{i+1}$ for $n \geq n_{0}$ for any given $x \in A_{i}$.

Proof (outline of proof). It follows directly by replacing (37) with

$$
\begin{aligned}
& d\left(T^{(n+1) p} x, T^{(n+1) p-1} x\right) \\
& \leq \widehat{K}^{(n)}(x, T x) d(T x, x)+\left(1-\widehat{K}^{(n)}(x, T x)\right) D, \\
& \forall x \in A_{i}, \quad \forall i \in \bar{p}, \quad \forall j \in \overline{p-1} \cup\{0\},
\end{aligned}
$$

so that there is the $\operatorname{limit}_{\lim _{n \rightarrow \infty}} d\left(T^{(n+1) p} x, T^{(n+1) p-1} x\right)=$ $0 ; \forall x \in A_{i}, \forall i \in \bar{p}$, and $\forall j \in \overline{p-1} \cup\{0\}$.

\section{Convergence of the Iterations to Best Proximity Points and Fixed Points}

Important results about convergence of iterated sequences of 2-cyclic self-mappings to unique best proximity points were firstly stated and proven in [11] and then widely used in the literature. Some of them are quoted here to be then used in the context of this paper. Consider a metric space $(X, d)$ with nonempty subsets $A, B \subset X$ such that $D=d(A, B) \geq 0$. The following basic results have been proven in the existing background literature.

Result 1 (see [11]). Let $(X, d)$ be a metric space, and let $A$ and $B$ be subsets of $X$. Then, if $A$ is compact and $B$ is approximatively compact with respect to $A$ (i.e., $d\left(y, x_{n}\right) \rightarrow d(y, B)$ as $n \rightarrow \infty$ for each sequence $\left\{x_{n}\right\}_{n \in \mathbf{Z}_{0+}} \subset \mathrm{B}$ for some $y \in$ $A)$, then $A^{o}=\left\{x \in A: d\left(x, y^{\prime}\right)=D\right.$ for some $\left.y^{\prime} \in B\right\}$ and $B^{o}=\left\{y \in B: d\left(x^{\prime}, y\right)=D\right.$ for some $\left.x^{\prime} \in A\right\}$ are nonempty.

It is known that if $A$ and $B$ are both compact, then $A$ (resp., $B$ ) is approximatively compact which respect to $B$ (resp., $A$ ).

Result 2 (see [11]). Let $(\mathrm{X},\|\|)$ be a reflexive Banach space, let $A$ be a nonempty, closed, bounded, and convex subset of $X$ and let $B$ be a nonempty, closed and convex subset of $X$. Then, the sets of best proximity points $A^{o}$ and $B^{o}$ are nonempty.

Result 3 (see [11]). Let $(X, d)$ be a metric space, let $A$ and $B$ be nonempty closed subsets of $X$, and let $T: A \cup B \rightarrow A \cup B$ be a 2-cyclic contraction. If either $A$ is boundedly compact (i.e., if any bounded sequence $\left\{x_{n}\right\}_{n \in Z_{0+}} \subset A$ has a subsequence converging to a point of $A$ ) or $B$ is boundedly compact, then there is $x \in A \cup B$ such that $d(x, T x)=D$.

Remark 11. It is known that if $A \subset X$ is boundedly compact, then it is approximatively compact. Also, a closed set $A$ of a normed space is boundedly compact if it is locally compact (the inverse is not true in separable Hilbert spaces [34]); equivalently, if and only if the closure of each bounded subset $C \subset A$ is compact and contained in $A$. If $(X, d)$ is a linear metric space, a closed subset $A \subset X$ is boundedly compact if each bounded $C \subset A$ is relatively compact. It turns out that if $A \subset X$ is closed and bounded then it is relatively compact [35]. It also turns out that if $(X, d)$ is a complete metric space and the metric is homogeneous and translation-invariant, then $(X, d)$ is a linear metric space and $(X,\|\|)$ is also a Banach space with \|\| being the norm induced by the metric $d$. Note that, since the metric is homogeneous and translation-invariant and since $(X, d)$ is a linear metric space, such a metric induces a norm. In such a Banach space, if $A \subset X$ is bounded and closed, then $A$ is boundedly compact and thus approximatively compact.

Result 4 (see $[11])$. Let $(X,\|\|)$ be a uniformly convex Banach space, let $A$ be a nonempty closed and convex subset of $X$, and let $B$ be a nonempty closed subset of $X$. Let sequences $\left\{x_{n}\right\}_{n \in \mathbf{Z}_{0+}} \subset A,\left\{z_{n}\right\}_{n \in \mathbf{Z}_{0+}} \subset A$ and $\left\{y_{n}\right\}_{n \in \mathbf{Z}_{0+}} \subset B$ satisfy $\| x_{n}-$ $y_{n} \| \rightarrow D$ and $\left\|z_{n}-y_{n}\right\| \rightarrow D$ as $n \rightarrow \infty$. Then $\left\|z_{n}-x_{n}\right\| \rightarrow$ 0 as $n \rightarrow \infty$. 
It is known that a uniformly convex Banach space $(X,\|\|)$ is reflexive and that a Banach space is a complete metric space $(X, d)$ with respect to the norm-induced distance.

Result 5 (see [11]). If $(X, d)$ is a complete metric space, $T$ : $A \cup B \rightarrow A \cup B$ is a 2 -cyclic contraction, where $A$ and $B$ are nonempty closed subsets of $X$, and the sequence $\left\{x_{n}\right\}_{n \in \mathbf{Z}_{0+}}$ generated as $x_{n+1}=T x_{n}, \forall n \in \mathbf{Z}_{+}$for a given $x_{0} \in A$ has a convergent subsequence $\left\{x_{2 n_{k}}\right\}_{n_{k} \in \mathbf{Z}_{0+}} \subset\left\{x_{2 n}\right\}_{n \in \mathbf{Z}_{0+}} \subset\left\{x_{n}\right\}_{n \in \mathbf{Z}_{0+}}$ in $A$, then there is $x \in A \cup B$ such that $d(x, T x)=D$.

Sufficiency-type results follow below concerning the convergence of iterated sequences being generated by contractive and strictly contractive $p$-semicyclic self-mappings, which are asymptotically $p$-cyclic, to best proximity or fixed points.

Theorem 12. Assume that $(X,\|\|)$ is a uniformly convex Banach space so that $(X, d)$ is a complete metric space ifd : $X \times$ $X \rightarrow \mathbf{R}_{0+}$ is the norm-induced metric. Assume, in addition, that $T: \bigcup_{i \in \bar{p}} A_{i} \rightarrow \bigcup_{i \in \bar{p}} A_{i}$ is a $p$-semicyclic impulsive selfmapping, where $A_{i} \subset X, \forall i \in \bar{p}$ are nonempty, closed, and convex subsets of $X$, and assume also that

(1) either the constraint (29), or the constraint (39) holds subject to (30) and (31) provided that the limit $\lim _{n \rightarrow \infty} \widehat{K}^{(n)}(x, T x)=0, \forall x \in \bigcup_{i \in \bar{p}} A_{i}$ exists and $m:\left(\bigcup_{i \in \bar{p}} A_{i}\right) \times\left(\bigcup_{i \in \bar{p}} A_{i}\right) \rightarrow \mathbf{R}_{0+}$ satisfies (35);

(2) for each given $x \in A_{i}$ for any $i \in \bar{p}$, there is a finite $k_{i}=$ $k_{i}(x) \in \mathbf{Z}_{0+}$ such that $\lim _{\inf _{n \rightarrow \infty}} T^{n p+k_{i}(x)} \in A_{i+1}$ (i.e., the p-semicyclic impulsive self-mapping $T$ : $\bigcup_{i \in \bar{p}} A_{i} \rightarrow \bigcup_{i \in \bar{p}} A_{i}$ is also an asymptotically $p$-cyclic one).

Then, $T: \bigcup_{i \in \bar{p}} A_{i} \rightarrow \bigcup_{i \in \bar{p}} A_{i}$ is either an asymptotically contractive or a strictly contractive $p$-semicyclic impulsive selfmapping, and, furthermore, the following properties hold.

(i) The limits below exist:

$$
\begin{aligned}
& \lim _{n \rightarrow \infty} d\left(T^{(n+1) p} x, T^{(n+j) p+j} x\right)=D, \\
& \forall x \in A_{i}, \quad \forall j \in \bar{k}_{i}, \quad \forall i \in \bar{p}, \\
& \lim _{n \rightarrow \infty} d\left(T^{(n+1) p+\bar{k}_{i}+1} x, T^{(n+j) p+\bar{k}_{i}} x\right)=0, \\
& \forall x \in A_{i}, \quad \forall i \in \bar{p},
\end{aligned}
$$

where $\bar{k}_{i}=\sup _{x \in A_{i}} k_{i}(x), \forall i \in \bar{p}$. Furthermore, $\left\{T^{n p} x\right\}_{n \in \mathbf{Z}_{+}} \rightarrow$ $z_{i},\left\{T^{n p+j} x\right\}_{n \in \mathbf{Z}_{+}} \rightarrow T z_{i}^{(j)}$ for any given $x \in A_{i}$ with $\left\{T^{n p+j} x\right\}_{n \in \mathbf{Z}_{+}} \subset A_{i} \cup A_{i+1}, \forall j \in \overline{\bar{k}}_{i}, \lim _{n \rightarrow \infty} T^{n p+\bar{k}_{i}} x \subset A_{i+1}$, $z_{i} \in A_{i}, z_{i}^{(j)} \in A_{i} ; \forall j \in \overline{\bar{k}}_{i}-1$, and $z_{i+1}=T z_{i}^{\left(k_{i}\right)} \in A_{i+1}$, $\forall i \in \bar{p}$. The points $z_{i}$ and $z_{i+1}$ are unique best proximity points in $A_{i}$ and $A_{i+1}, \forall i \in \bar{p}$ of $T: \bigcup_{i \in \bar{p}} A_{i} \rightarrow \bigcup_{i \in \bar{p}} A_{i}$, and there is a unique limiting set

$$
\begin{aligned}
& \left(z_{1}, z_{1}^{(1)}=T z_{1}, \ldots, z_{2}=z_{1}^{\left(\bar{k}_{1}\right)}=T^{\bar{k}_{1}} z_{1}, \ldots, z_{p}, z_{p}^{(1)}\right. \\
& \left.\quad=T z_{p}, \ldots, z_{p}^{\left(\bar{k}_{p}-1\right)}=T^{\bar{k}_{p}-1} z_{p}\right) \subset A_{1}^{\bar{k}_{1}} \times \cdots \times A_{1}^{\bar{k}_{p}} .
\end{aligned}
$$

If $\bigcap_{i \in \bar{p}} A_{i} \neq \emptyset$, then the $p$ best proximity points $z_{i}=z \in$ $\bigcap_{j \in \bar{p}} A_{j}, \forall i \in \bar{p}$ become a unique fixed point $z$ of $T$ : $\bigcup_{i \in \bar{p}} A_{i} \rightarrow \bigcup_{i \in \bar{p}} A_{i}$.

(ii) Assume that the constraint (15) holds, subject to either (25), or (29), with $\bar{K}=\prod_{i=1}^{p-1}\left[K_{i}\right]$ and $\widehat{K} \in[0,1)$ defined in (16). Assume, in addition, that for each $x \in A_{i}$ for any $i \in \bar{p}$, it exists a finite $k_{i}=k_{i}(x) \in \mathbf{Z}_{0+}$ such that $\lim \inf _{n \rightarrow \infty} T^{n p+k_{i}(x)} \in A_{i+1}$ with $\bar{k}_{i}=\sup _{x \in A_{i}} k_{i}(x), \forall i \in \bar{p}$. Then, Property (i) still holds.

Proof. The existence of the limits (41) and (42) follows from (34) in Theorem 9 and the above background Result 4 [11] since, for each $x \in A_{i}$ for any $i \in \bar{p}$, there is a finite $k_{i}=k_{i}(x) \in \mathbf{Z}_{0+}$ such that $\lim \inf _{n \rightarrow \infty} T^{n p+k_{i}(x)} \in A_{i+1}$ with $\bar{k}_{i}=\sup _{x \in A_{i}} k_{i}(x), \forall i \in \bar{p}$ so that the limits (41) exist (note that $\bar{k}_{i}=1, \forall i \in \bar{p}$ if $T: \bigcup_{i \in \bar{p}} A_{i} \rightarrow \bigcup_{i \in \bar{p}} A_{i}$ is a $p^{-}$ cyclic impulsive self-mapping). The limit (42) exists from the background Results 1 and 5 of [11] with $z_{i} \in A_{i}$ and $z_{i+1}=T z_{i}^{\left(k_{i}\right)} \in A_{i+1}, \forall i \in \bar{p}$ being unique best proximity points of $T: \bigcup_{i \in \bar{p}} A_{i} \rightarrow \bigcup_{i \in \bar{p}} A_{i}$ in $A_{i}$ and $A_{i+1} ; \forall i \in \bar{p}$ since $(X, d)$ is also a $(X,\|\|)$ uniformly convex Banach space for the norm-induced metric and the subsets $A_{i}$ of $X, \forall i \in \bar{p}$ are nonempty, closed and convex. The limiting set $\left(z_{i}, z_{i}^{(1)}=\right.$ $\left.T z_{i}, \ldots, z_{i+1}=T^{\bar{k}_{i}} z_{i}\right)$ is unique with $z_{i}^{(j)} \in A_{i} ; \forall j \in \overline{\bar{k}}_{i}-1$ since $z_{i}$ and $z_{i+1} ; \forall i \in \bar{p}$ are unique best proximity points and $T$ : $\bigcup_{i \in \bar{p}} A_{i} \rightarrow \bigcup_{i \in \bar{p}} A_{i}$ is single-valued. Property (i) has been proved. The same conclusions arise from (25) in Corollary 6 and from (39) in Corollary 10 leading to Property (ii).

Remarks 13. (1) Note that if the self-mapping $T: \bigcup_{i \in \bar{p}} A_{i} \rightarrow$ $\bigcup_{i \in \bar{p}} A_{i}$ is an asymptotic $p$-cyclic impulsive one, then the limiting set (43) of Theorem 12 can only contain points which are not best proximity points in bounded subsets $A_{i}$ of $X$ whose diameter is not smaller than $D$.

(2) Under the conditions of Theorem 12, if $T: \bigcup_{i \in \bar{p}} A_{i} \rightarrow$ $\bigcup_{i \in \bar{p}} A_{i}$ is, in particular, a contractive or strictly contractive $p$-cyclic impulsive self-mapping, then the limiting set (43) only contains best proximity points; that is, it is of the form $\left(z_{1}, z_{2}, \ldots, z_{p}\right)$. If $\bigcap_{i \in \bar{p}} A_{i} \neq \emptyset$, then such a set reduces to a unique best proximity point $z \in \bigcap_{i \in \bar{p}} A_{i}$.

(3) Note that Theorem 12 can be formulated also for a complete metric space $(X, d)$ with a homogeneous translation-invariant metric $d: X \times X \rightarrow \mathbf{R}_{0+}$ being equivalent to a Banach space $(X,\|\|)$, where \|\| is the metricinduced norm, which is uniformly convex so that it is also a complete. Note that such a statement is well-posed since a norm-induced metric exists if such a metric is homogeneous and translation invariant. 
It turns out that Theorem 12 and Remarks 13 also hold if $T: \bigcup_{i \in \bar{p}} A_{i} \rightarrow \bigcup_{i \in \bar{p}} A_{i}$ is either a contractive or a strictly contractive $p$-semicyclic impulsive self-mapping as stated in the subsequent result.

Corollary 14. Theorem 12 holds, in particular, if $T$ : $\bigcup_{i \in \bar{p}} A_{i} \rightarrow \bigcup_{i \in \bar{p}} A_{i}$ is a contractive or strictly contractive p-semicyclic impulsive self-mapping with $K_{i}=K \in[0,1)$, $\forall i \in \bar{p}$ being a constant in (29) or (39) subject to (35) and $m:\left(\bigcup_{i \in \bar{p}} A_{i}\right) \times\left(\bigcup_{i \in \bar{p}} A_{i}\right) \rightarrow \mathbf{R}_{0+}$ being not larger than unity.

Theorem 12 also holds if $T: \bigcup_{i \in \bar{p}} A_{i} \rightarrow \bigcup_{i \in \bar{p}} A_{i}$ is, in particular, a contractive or strictly contractive p-cyclic impulsive self-mapping with $K_{i}=K \in[0,1) ; \forall i \in \bar{p}$ being constant in (29) or (39) subject to (35) and $m:\left(\bigcup_{i \in \bar{p}} A_{i}\right) \times$ $\left(\bigcup_{i \in \bar{p}} A_{i}\right) \rightarrow \mathbf{R}_{0+}$ being not larger than unity. In this case, the limiting set (43) only contains best proximity points; that is, it is of the form $\left(z_{1}, z_{2}, \ldots, z_{p}\right)$.

\section{Application Examples to Impulsive Differential and Difference Equations}

Recent results about best proximity points concerning psiGeraghty contractions and on cyclic orbital contractions are obtained in $[36,37]$, respectively. On the other hand, it turns out that fixed point theory is a useful tool to study the stability of differential and difference equations and dynamic systems [38-42]. Some worked examples are given in the sequel concerning the global feedback stabilization and the stability of the equilibrium points [43-46], linked with fixed points and best proximity points of impulsive and timedelayed differential equations. The subsequent examples rely on the properties of iterated sequences $x_{n+1}=T x_{n}, \forall n \in \mathbf{Z}_{0+}$ for any $x_{0} \in \bigcup_{i \in \bar{p}} A_{i}$ being generated from nonexpansive or contractive $p$-semicyclic, impulsive self-mappings $T A_{i} \rightarrow$ $A_{i} \cup A_{i+1}$, where $A_{i} \subset X, \forall i \in \bar{p}$ and $(X, d)$ is a metric space, subject to theb following:

(1) $T^{-}: \bigcup_{i \in \bar{p}} A_{i} \rightarrow \bigcup_{i \in \bar{p}} A_{i}$ is a nonexpansive, or a contractive, $p$-semicyclic self-mapping so that $T^{-} A_{i} \subseteq A_{i} \cup A_{i+1}, \forall i \in \bar{p}$ subject to the cyclic nonexpansive/contractive constraint $d\left(T^{-} x, T^{-} y\right) \leq$ $K d(x, y)+(1-K) D, \forall i \in \bar{p}$ for $K \in[0,1]$, where $D$ is the distance between any two adjacent subsets. This self-mapping describes in the given examples the discretized impulsive-free solution of an ordinary differential equation;

(2) $T^{+}: \bigcup_{i \in \bar{p}} A_{i} \rightarrow \bigcup_{i \in \bar{p}} A_{i}$ satisfies a distance discontinuity condition of the type $d\left(T^{+}\left(T^{-} x\right), T^{+}\left(T^{-} y\right)\right) \leq$ $m\left(T^{-} x, T^{-} y\right) d\left(T^{-} x, T^{-} y\right)$ for some given bounded function $m:\left(\bigcup_{i \in \bar{p}} A_{i}\right) \times\left(\bigcup_{i \in \bar{p}} A_{i}\right) \rightarrow \mathbf{R}_{0+}$. This selfmapping describes the bounded steps in the solution due to eventual forcing impulses at certain impulsive time instants.
Example 15. Consider the real impulsive differential equation

$$
\begin{gathered}
\dot{x}(t)=\alpha(t) \quad x(t)+\beta(t) u(t)+\sum_{t_{k} \in \mathbf{S I}} \gamma\left(t_{k}\right) \delta\left(t-t_{k}\right), \\
x\left(0^{-}\right)=x_{0}, \quad x(0)=x\left(0^{+}\right)=x_{0}+\gamma(0),
\end{gathered}
$$

where $\alpha, \beta \in B\left(\mathbf{R}_{0+}, \mathbf{R}\right) \cup \operatorname{PC}\left(\mathbf{R}_{0+}, \mathbf{R}\right)$ (i.e., bounded and piece-wise continuous real functions on $\left.\mathbf{R}_{0+}\right),\left\{\gamma\left(t_{k}\right)\right\}_{t_{k} \in \mathbf{S I}}$ is a bounded sequence, $\delta(t)$ is the Dirac distribution, $u \in$ $\operatorname{PC}\left(\mathbf{R}_{0+}, \mathbf{R}\right)$ is the nonimpulsive control, $x: \mathbf{R}_{0+} \rightarrow \mathbf{R}$ is the unique solution of (44) which is continuous and timedifferentiable on $\left[0, t_{1}\right) \bigcup\left(\bigcup_{k \in \mathbf{I}}\left[t_{k}, t_{k+1}\right)\right), t_{k} \in \mathbf{S I} \subset \mathbf{R}_{0+}$ is a set of impulsive sampling instants with $k \in \mathbf{I} \subseteq \mathbf{Z}_{+}$, and the indicator set $\mathbf{I}$ of $\mathbf{S I}$ has a finite or an infinite cardinal. Note that $u_{\text {imp }}(t)=\sum_{t_{k} \in \mathrm{SI}} \gamma\left(t_{k}\right) \delta\left(t-t_{k}\right)$ is an impulsive control. Assume a linear-feedback control of the form $u(t)=g(t) x(t)$ with $g \in \mathrm{PC}\left(\mathbf{R}_{0+}, \mathbf{R}\right)$. The solution of (44) is

$$
\begin{aligned}
& x(t)=e^{\int_{t_{k}}^{t} \alpha(\tau) d \tau} x\left(t_{k}\right)+\int_{t_{k}}^{t} e^{\int_{\tau}^{t} \alpha(\sigma) d \sigma} \beta(\tau) u(\tau) d \tau \\
&=e^{\int_{t_{k}}^{t}(\alpha(\tau)+\beta(\tau) g(\tau)) d \tau} x\left(t_{k}\right), \\
& \forall t \in\left(\left[0, t_{1}\right) \bigcup\left(\bigcup_{t_{k} \in \mathbf{S I}}\left[t_{k}, t_{k+1}\right)\right)\right), \\
& x\left(t_{k+1}\right):=x\left(t_{k+1}^{+}\right)=x\left(t_{k+1}^{-}\right)+\gamma\left(t_{k}\right) \\
&=e^{\int_{t_{k}}^{t}(\alpha(\tau)+\beta(\tau) g(\tau)) d \tau} x\left(t_{k}\right)+\gamma\left(t_{k+1}\right) \\
&=e^{\int_{t_{k}}^{t_{k+1}} \alpha(\tau) d \tau} x\left(t_{k}\right)+\int_{t_{k}}^{t_{k+1}} e^{\int_{\tau}^{t_{k+1}} \alpha(\sigma) d \sigma} \beta(\tau) u(\tau) d \tau \\
&=e^{\int_{t_{k}}^{t_{k+1}}(\alpha(\tau)+\beta(\tau) g(\tau)) d \tau} x\left(t_{k}\right)+\gamma\left(t_{k+1}\right) ; \quad \forall t_{k} \in \mathbf{S I} .
\end{aligned}
$$

Then, the following results hold.

Proposition 16. Assume that $\gamma(0)=M(0), \gamma\left(t_{k+1}\right)=M\left(t_{k+1}\right)$ $-e^{\int_{t_{k}}^{t_{k}+1}(\alpha(\tau)+\beta(\tau) g(\tau)) d \tau} x\left(t_{k}\right)$ for $t_{k} \in \mathbf{S I}$ and $\left\{M\left(t_{k}\right)\right\}_{t_{k} \in \mathbf{S I}}$ is some bounded real sequence, then

(i) $\left\{x\left(t_{k}\right)\right\}_{t_{k} \in \mathbf{S I}}$ is bounded so that (44) is globally stable. If, in addition, $\left\{M\left(t_{k}\right)\right\}_{t_{k} \in S I}$ converges to zero as $t_{k}(\epsilon$ SI $\rightarrow \infty$ (if $c=$ card $\mathbf{I}$ is finite then $M\left(t_{c}\right)=0$ ), then $\left\{x\left(t_{k}\right)\right\}_{t_{k} \in \mathbf{S I}}$ converges to zero as $k \rightarrow \infty$.

(ii) if $\mathbf{I}$ has an infinite cardinal, so that $\mathbf{I}=\mathbf{Z}_{+}$, and $\mid t_{k+1}-$ $t_{k} \mid \leq \bar{T}<\infty, \forall t_{k} \in \mathbf{S I}$, then $|x(t)|$ is bounded for all $t \in$ $\mathbf{R}_{0+}$. If, in addition, $\left\{M\left(t_{k}\right)\right\}_{t_{k} \in \mathbf{S I}}$ converges to the stable zero equilibrium point as $k \rightarrow \infty$ then $x(t) \rightarrow 0$ as $t \rightarrow \infty$ so that (44) is globally asymptotically stable.

Proof. Property (i) follows from its statement and (46). Since $\left\{x\left(t_{k}\right)\right\}_{t_{k} \in \mathbf{S I}}$ is bounded, the continuous function $x(t)$ on $\left(t_{k}, t_{k+1}\right)$ cannot be unbounded on the finite interval $\left[t_{k}, t_{k+1}\right)$ if $0 \leq \bar{T}_{0} \leq\left|t_{k+1}-t_{k}\right| \leq \bar{T}<\infty, \forall t_{k} \in$ SI. 
Since $c l \mathbf{R}_{0+}=c l\left(\left[0, t_{1}\right) \bigcup\left(\bigcup_{k \in \mathbf{I}}\left[t_{k}, t_{k+1}\right)\right)\right)$ if the indicator set I of impulses is of infinite cardinal, it becomes obvious that $x(t)$ is bounded on its definition domain $\mathbf{R}_{0+}$. If, in addition, $\left\{M\left(t_{k}\right)\right\}_{t_{k} \in \mathbf{S I}}$ converges to zero as $k \rightarrow \infty$ then $\left\{x\left(t_{k}\right)\right\}_{t_{k} \in \mathbf{S I}}$ converges to zero as $k \rightarrow \infty$ from Property (i) so that $\left\{\gamma\left(t_{k}\right)\right\}_{t_{k} \in \mathrm{SI}}$ converges to zero as $k \rightarrow \infty$. Then, $x(t) \rightarrow 0$ on $t \in\left[t_{k}, t_{k+1}\right)$ as $t_{k}(\in \mathbf{S I}) \rightarrow \infty$ from (45). Hence, Property (ii) is proven.

Proposition 17. Assume that $\gamma\left(t_{k}\right)=\lambda\left(t_{k}\right) x\left(t_{k}^{-}\right)$. Then, the following properties hold.

(i) Assume that card $\mathbf{S I}=\chi_{0}$ (i.e., the infinity cardinal of a numerable set) fulfilling $\left|t_{k+1}-t_{k}\right| \leq \bar{T}<\infty, \forall t_{k} \in \mathbf{S I}$, and define the self-mapping $T: \mathbf{R} \rightarrow \mathbf{R}$ generating the solution sequence $\left\{x\left(t_{k}\right)\right\}_{t_{k} \in \mathrm{SI}}$ of (44) at the set of impulsive time instants $\mathbf{S I}=\left\{t_{k}\right\}_{k \in \mathbf{I}}$. Assume that such a set has infinite cardinal. Then, $T: \mathbf{R} \rightarrow \mathbf{R}$ is asymptotically contractive and has a unique fixed point $\bar{x}=0$ if

$$
0 \leq \lim _{\mathbf{S I} \ni t_{k} \rightarrow \infty} \sup _{p}\left|1+\lambda\left(t_{k+1}\right)\right| e^{\int_{t_{k}}^{t_{k+1}}(\alpha(\tau)+\beta(\tau) g(\tau)) d \tau}<1 .
$$

If card $\mathbf{I}=c<\chi_{0}$ then $(47 \mathbf{a})$ is replaced with

$$
\begin{aligned}
0 & \leq \limsup _{t \rightarrow \infty} e^{\int_{t_{c}}^{t}(\alpha(\tau)+\beta(\tau) g(\tau)) d \tau} \\
& <1 \Longleftrightarrow \lim _{t \rightarrow \infty} \sup _{t \rightarrow \infty} \int_{t_{c}}^{t}(\alpha(\tau)+\beta(\tau) g(\tau)) d \tau<0 .
\end{aligned}
$$

Furthermore, $\left\{x\left(t_{k}\right)\right\}_{t_{k} \in \mathbf{S I}}$ is bounded, $x: \mathbf{R}_{0+} \rightarrow \mathbf{R}$ is bounded and $\lim _{t \rightarrow \infty} x(t)=0$ so that (44) is globally asymptotically stable.

(ii) Property (i) still holds if card $\mathbf{S I}=\chi_{0}$, and there is a nondecreasing sequence $\left\{N_{k}\right\}_{k \in \mathbf{Z}_{0+}} \subseteq$ I with $\left|N_{k+1}-N_{k}\right| \leq \bar{N}<$ $\infty$ such that

$$
\lim _{\mathbf{I} \ni N_{k} \rightarrow \infty}\left|\prod_{i=N_{k}}^{N_{k+1}}\left[\left(1+\lambda\left(t_{i}\right)\right) e^{\int_{t_{i}}^{t_{i+1}}(\alpha(\tau)+\beta(\tau) g(\tau)) d \tau}\right]\right|<1 .
$$

(iii) Property (i) also holds with $T: \mathbf{R} \rightarrow \mathbf{R}$ being contractive if card $\mathbf{S I}=\chi_{0}$ and for some positive real sequence $\left\{\varepsilon_{k}\right\}_{k \in \mathbf{Z}_{0+}}$

$$
\begin{array}{r}
\lambda\left(t_{k+1}\right)=e^{-\int_{t_{k}}^{t_{k+1}}(\alpha(\tau)+\beta(\tau) g(\tau)) d \tau}-1-\varepsilon_{k} \\
\forall k \in \mathbf{Z}_{0+}
\end{array}
$$

Proof. Assume that card $\mathbf{S I}=\chi_{0}$. It follows that

$$
\begin{aligned}
& x\left(t_{k+1}\right)=\left(1+\lambda\left(t_{k+1}\right)\right) x\left(t_{k+1}^{-}\right) \\
&=\left(1+\lambda\left(t_{k+1}\right)\right) e^{\int_{t_{k}}^{t_{k+1}}(\alpha(\tau)+\beta(\tau) g(\tau)) d \tau} x\left(t_{k}\right), \\
& \forall t_{k} \in \mathbf{S I},
\end{aligned}
$$

so that

$$
\begin{aligned}
& \left|x\left(t_{k+1}\right)-x\left(t_{k}\right)\right| \\
& \leq \sup _{t_{k} \in \mathbf{S I}}\left(\left|1+\lambda\left(t_{k+1}\right)\right| e^{\int_{t_{k}}^{t_{k+1}}(\alpha(\tau)+\beta(\tau) g(\tau)) d \tau}\right) \\
& \quad \times\left|x\left(t_{k}\right)-x\left(t_{k-1}\right)\right|, \quad \forall t_{k} \in \mathbf{S I}
\end{aligned}
$$

and one gets that $\left\{\left|x\left(t_{k+1}\right)-x\left(t_{k}\right)\right|\right\}_{t_{k} \in \mathbf{S I}}$ converges to zero and $\left\{x\left(t_{k}\right)\right\}_{t_{k} \in \mathbf{S I}}$ converges to a unique fixed point $\bar{x} \epsilon$ $\mathbf{R}$ as SI $\ni \quad t_{k} \rightarrow \infty$ from Theorem 12, supported by Theorem 9, with the complete metric space and Banach space $\left(\mathbf{R}_{0+}, d\right) \equiv\left(\mathbf{R}_{0+},\|\|\right)$ the metric being the Euclidean distance. Also, since the sequence $\left\{\left|x\left(t_{k+1}\right)-x\left(t_{k}\right)\right|\right\}_{t_{k} \in \mathrm{SI}}$ converges to zero as SI $\ni t_{k} \rightarrow \infty$ yields that $\bar{x}=0$ is the unique fixed point of $T: \mathbf{R} \rightarrow \mathbf{R}$ since, otherwise, (50) would contradict (47a) for $\bar{x}=x\left(t_{k}\right)=x\left(t_{k+1}\right) \neq 0$. The facts that $\left\{x\left(t_{k}\right)\right\}_{t_{k} \in \mathbf{S I}}$ is bounded, $x: \mathbf{R}_{0+} \rightarrow \mathbf{R}$ is bounded, and $\lim _{t \rightarrow \infty} x(t)=0$ follow under the same reasoning as in Proposition 16. Hence, Property (i) follows for the case that card $\mathbf{S I}=\chi_{0}$. If such a cardinal is finite, we can remove a finite number of impulsive time instants from the discussion, and the property also holds under (47b). The proof of Property (ii) is similar leading to the convergence to zero of the sequence $\left\{\delta \widehat{x}\left(t_{N_{k}}\right)\right\}_{t_{N_{k}} \in \overline{\mathbf{S I}} \subseteq \mathbf{S I}}$ as $t_{N_{k}} \rightarrow \infty$ where $\delta \widehat{x}\left(t_{N_{k}}\right)=x\left(t_{N_{k+1}}\right)-$ $x\left(t_{N_{k}}\right)$. Thus, $x\left(t_{N_{k}}\right) \rightarrow \hat{x}$ as $\overline{\mathbf{I}} \ni N_{k} \rightarrow \infty$ where $\overline{\mathbf{I}} \subseteq \mathbf{I}$. As above, it turns out that $\widehat{x}=\bar{x}=0$ under a similar contradiction argument to the above one. Hence, Property (ii) follows. Property (iii) follows directly since (49) leads to (47a).

Note that Proposition 17 states global properties for the solution so that the contractive condition is achievable with mixed conditions on the nonimpulsive and impulsive parts of the differential equation. For instance, it is clear from (49) that a certain condition on the impulsive controls can stabilize the system even if the nonimpulsive part is unstable; that is, if $\lim \inf _{\text {SI } \ni t_{k} \rightarrow \infty} \int_{t_{k}}^{t_{k+1}}(\alpha(\tau)+\beta(\tau) g(\tau)) d \tau>0$. It is easy to deduce from a slightly extended Proposition 17 that $T: \mathbf{R} \rightarrow$ $\mathbf{R}$ is asymptotically nonexpansive if the inequalities in (47a)(48) are not strict. Note also that (49) can be checked in terms of the values of intervals in-between consecutive impulsive time instants and impulsive control gains with the following test if SI has infinite cardinal:

$$
\begin{array}{r}
\ln \left|1+\lambda\left(t_{k+1}\right)\right| \\
+\int_{0}^{t_{k+1}-t_{k}}\left(\alpha\left(t_{k}+\tau\right)+\beta\left(t_{k}+\tau\right) g\left(t_{k}+\tau\right)\right) d \tau<0, \\
\forall t_{k} \in \mathbf{S I} .
\end{array}
$$

Then, the mapping constructing the solution which iterates at the impulsive time instants is contractive. The above 
closed-loop stability condition (52) is guaranteed if $\mid t_{k+1}-$ $t_{k} \mid \leq \bar{T}<\infty, \forall t_{k} \in$ SI and

$$
\begin{aligned}
\sup _{t_{k} \in \mathbf{S I}}\left[\ln \left|1+\lambda\left(t_{k+1}\right)\right|\right. \\
\left.\quad+\sup _{\tau \in[0, \bar{T}]}\left(\alpha\left(t_{k}+\tau\right)+\beta\left(t_{k}+\tau\right) g\left(t_{k}+\tau\right)\right) \bar{T}\right]<0 .
\end{aligned}
$$

Related close conditions to (52) and (53) would follow being equivalent to (47a) and (48) to guarantee that the mapping building the solution sequence at impulsive time instants from any initial condition is asymptotically contractive. In particular, a close test can be jointly performed for finite sets of consecutive impulsive time instants defined bounded time intervals. Closed-loop global asymptotic stability of the feedback equation and the convergence to the unique equilibrium point $\bar{x}=0$ is also guaranteed by the subsequent result.

Proposition 18. Assume that card $\mathbf{S I}=\chi_{0}$ and that there is a real sequence $\left\{q\left(t_{k}\right)\right\}_{k \in \mathbf{Z}_{0+}}$ fulfilling $0 \leq q\left(t_{k}\right)<1 ; \forall t_{k} \in \mathbf{S I}$ such that $\left\{\gamma\left(t_{k}\right)\right\}_{k \in \mathbf{Z}_{0+}}$ in (44) is defined by:

$$
\gamma\left(t_{k+1}\right)=-\left(q\left(t_{k}\right)+e^{\int_{t_{k}}^{t_{k+1}}(\alpha(\tau)+\beta(\tau) g(\tau)) d \tau}\right) x\left(t_{k}\right)
$$

$\forall k \in \mathbf{S I}$.

Then, $x\left(t_{k}\right) \rightarrow 0$ as $t_{k} \rightarrow \infty$ and $x(t) \rightarrow \bar{x}=0$ (the unique fixed point of $T: \mathbf{R} \rightarrow \mathbf{R}$ ) as $\rightarrow \infty$.

Proof. It follows from (45) by noting that (54) is equivalent to

$$
\begin{aligned}
x\left(t_{k+1}\right) & =e^{\int_{t_{k}}^{t_{k+1}}(\alpha(\tau)+\beta(\tau) g(\tau)) d \tau} x\left(t_{k}\right)+\gamma\left(t_{k+1}\right) \\
& =-q\left(t_{k}\right) x\left(t_{k}\right) .
\end{aligned}
$$

Example 19. Consider the differential equation (44) and the sets $A=\{z \in \mathbf{R}: z \leq-D / 2\}$ and $B=\{z \in \mathbf{R}: z \geq D / 2\}$ for some real $D \in \mathbf{R}_{0+}$. Define the self-mapping $T: \mathbf{R} \rightarrow \mathbf{R}$ for the solution sequence at impulsive time instants as follows for each $t_{k} \in \mathbf{S I}$ assuming that card $\mathbf{S I}=\chi_{0}$ and that there are prefixed finite $\bar{T}>0$ and $\bar{T}_{0}>0$ with $0<\bar{T}_{0} \leq\left|t_{k+1}-t_{k}\right| \leq \bar{T}$.

(a) $\lambda\left(t_{k+1}\right)$ and $t_{k+1} \leq t_{k}+\bar{T}$ are chosen so that

$$
\lambda\left(t_{k+1}\right)=-\left(1+e^{\int_{t_{k}}^{t_{k+1}}(\alpha(\tau)+\beta(\tau) g(\tau)) d \tau}\right)
$$

if $x\left(t_{k}\right) \leq-D / 2$ (i.e., if $x\left(t_{k}\right) \in A$ ) leading to $x\left(t_{k+1}\right) \geq D / 2$ (i.e., $x\left(t_{k+1}\right) \in B$ ) or if $\min \left(x\left(t_{k-1}\right), x\left(t_{k}\right)\right) \geq D / 2$ (i.e., if $x\left(t_{k-1}\right), x\left(t_{k}\right) \in B$ ) leading to $x\left(t_{k+1}\right) \leq-D / 2$ (i.e., $x\left(t_{k+1}\right) \in$ $A)$; and (b) $\lambda\left(t_{k+2}\right)$ and $t_{k+2} \leq t_{k+1}+\bar{T}$ are chosen so that for some given positive real constant $\mu$ :

$$
\begin{aligned}
& \ln \left|1+\lambda\left(t_{k+2}\right)\right| \\
& +\sup _{\tau \in\left[0, t_{k+2}-t_{k+1}\right]}\left(\alpha\left(t_{k+1}+\tau\right)+\beta\left(t_{k+1}+\tau\right) g\left(t_{k+1}+\tau\right)\right) \\
& \quad \times\left(t_{k+2}-t_{k+1}\right)=-\mu_{k+1} \leq-\mu<0
\end{aligned}
$$

leading to $x\left(t_{k+2}\right) \geq D / 2$ if $x\left(t_{k}\right) \leq-D / 2$ and $x\left(t_{k+1}\right) \geq D / 2$ (i.e., if $x\left(t_{k}\right) \in A$ and $x\left(t_{k+1}\right) \in B$ ) and leading to $x\left(t_{k+2}\right) \leq$ $-D / 2$ if $x\left(t_{k}\right) \geq D / 2$ and $x\left(t_{k+1}\right) \leq-D / 2$ (i.e., if $x\left(t_{k}\right) \in B$ and $\left.x\left(t_{k+1}\right) \in A\right)$.

Note that (56a) implies that $\gamma\left(t_{k+1}\right)=(1+$ $\left.e^{\int_{t_{k}}^{t_{k+1}}(\alpha(\tau)+\beta(\tau) g(\tau)) d \tau}\right)\left|x\left(t_{k}\right)\right|$ and $x\left(t_{k+1}\right)=-x\left(t_{k}\right)$. Note that (56b) leads to the strict contraction (39) of Corollary 10 in the particular form

$$
\begin{aligned}
& x\left(t_{k+2}\right)-D \\
& \quad=\left(1+\lambda\left(t_{k+2}\right)\right) e^{\int_{t_{k+1}}^{t_{k+2}}(\alpha(\tau)+\beta(\tau) g(\tau)) d \tau}\left(x\left(t_{k+1}\right)-D\right) .
\end{aligned}
$$

Note that (56a) and (56b) imply that the sequence of iterates is formed with consecutive sets of two consecutive points in $B$ and one in $A$. Thus, the sequence of impulsive gains (56a) and (56b) implies that the self-mapping $T: A \cup B \rightarrow A \cup B$, which generates the sequence $\left\{x_{t_{k}}\right\}_{t_{k} \in \mathbf{S I}}$, which is bounded, is $p$-semicyclic nonexpansive, while the composite self-mapping $T^{2}: A \cup B \rightarrow A \cup$ $B$, which generates the bounded subsequences $\left\{x_{t_{2 k}}\right\}_{t_{2 k} \in \mathbf{S I}}$ and $\left\{x_{t_{2 k+1}}\right\}_{t_{2 k+1} \in \mathbf{S I}}$, is 2-semicyclic contractive. As a result, $\left\{x_{t_{2 k+1}}\right\}_{t_{2 k} \in \mathrm{SI}}$ and $\left\{x_{t_{2 k+1}}\right\}_{t_{2 k+1} \in \mathrm{SI}}$ converge each to one of the unique best proximity points $\pm D / 2$, in particular, to the unique fixed point $\bar{x}=0$ if $D=0$.

Example 20. The differential equation (44) is now replaced by the functional impulsive differential equation with delay $h>0$ as follows:

$$
\begin{aligned}
\dot{x}(t)= & \alpha(t) x(t)+\alpha_{0}(t) x(t-h)+\beta(t) u(t) \\
& +\sum_{t_{k} \in S I} \gamma\left(t_{k}\right) \delta\left(t-t_{k}\right)
\end{aligned}
$$

with $\alpha_{0} \in B\left(\mathbf{R}_{0+}, \mathbf{R}\right) \cup \operatorname{PC}\left(\mathbf{R}_{0+}, \mathbf{R}\right)$ and $\varphi:[-h, 0] \rightarrow$ $\mathbf{R}$ being any absolutely continuous of initial conditions of (58) with eventual bounded discontinuities on a subset of $[-h, 0]$ of zero measure with $\varphi\left(0^{-}\right)=x\left(0^{-}\right)=x_{0}$ and $\varphi\left(0^{+}\right)=x\left(0^{+}\right)=\varphi(0)=x_{0}+\gamma(0)$ so that $x(t)=\varphi(t), t \in$ $[-h, 0]$. Thus, the solution of (58) is unique and continuous 
and differentiable in $\left[0, t_{1}\right) \cup\left(\bigcup_{k \in \mathbf{I}}\left[t_{k}, t_{k+1}\right)\right)$. The unique solution of (58) is

$$
\begin{aligned}
x(t)= & \Psi(t, 0) x(0)+\int_{0}^{h} \Psi(t, \tau) \varphi(\tau-h) d \tau \\
& +\int_{0}^{t} \Psi(t, \tau) u(\tau) d \tau+\sum_{t_{k} \in \mathbf{S I}} \Psi\left(t, t_{k}\right) \gamma\left(t_{k}\right) \mathbf{1}\left(t-t_{k}\right),
\end{aligned}
$$$$
\forall t \in \mathbf{R}_{0+} \text {, }
$$$$
x\left(t_{k+1}\right)=\Psi\left(t_{k+1}, t_{k}\right) x\left(t_{k}\right)
$$$$
\begin{aligned}
& +\int_{0}^{h} \Psi\left(t_{k+1}, t_{k}+\tau\right) x\left(t_{k}+\tau-h\right) d \tau \\
& +\int_{0}^{t_{k+1}-t_{k}} \Psi\left(t_{k+1}, t_{k}+\tau\right) \beta\left(t_{k}+\tau\right) u\left(t_{k}+\tau\right) d \tau \\
& +\gamma\left(t_{k+1}\right) .
\end{aligned}
$$

where $\mathbf{1}(t)$ is the unit step (Heaviside) function, where the evolution operator satisfies

$$
\dot{\Psi}(t, \tau)=\alpha(t) \Psi(t, \tau)+\alpha_{0}(t) \Psi(t-h, \tau),
$$

for $t \geq \tau$ with initial conditions $\Psi(0)=1, \Psi(t)=0$ for $t<0$. Thus, (60) has the unique continuously differentiable solution

$\Psi(t, 0)$

$$
\begin{array}{r}
=e^{\int_{0}^{t} \alpha(\tau) d \tau}+\int_{h}^{t} e^{\int_{\tau}^{t} \alpha(\sigma) d \sigma} \alpha_{0}(\tau) \Psi(\tau-h, 0) d \tau, \\
\forall t \in \mathbf{R}_{0+} .
\end{array}
$$

If $t_{i} \in \mathrm{SI}$ then (60) has the unique solution at $t=t_{i}$ as follows:

$$
\begin{aligned}
x\left(t_{i}^{-}\right)= & \Psi\left(t_{i}, 0\right) x(0)+\int_{0}^{h} \Psi\left(t_{i}, \tau\right) \varphi(\tau-h) d \tau \\
& +\int_{0}^{t_{i}} \Psi\left(t_{i}, \tau\right) u(\tau) d \tau \\
& +\sum_{t_{k}\left(<t_{i}\right) \in \mathrm{SI}} \Psi\left(t_{i}, t_{k}\right) \gamma\left(t_{k}\right) \mathbf{1}\left(t_{i}-t_{k}\right), \\
x\left(t_{i}\right)= & \Psi\left(t_{i}, 0\right) x(0)+\int_{0}^{h} \Psi\left(t_{i}, \tau\right) \varphi(\tau-h) d \tau \\
& +\int_{0}^{t_{i}} \Psi\left(t_{i}, \tau\right) u(\tau) d \tau \\
& +\sum_{t_{k}\left(\leq t_{i}\right) \in \mathrm{SI}} \Psi\left(t_{i}, t_{k}\right) \gamma\left(t_{k}\right) \mathbf{1}\left(t_{i}-t_{k}\right) \\
= & x\left(t_{i}^{-}\right)+\gamma\left(t_{i}\right) .
\end{aligned}
$$

Equation (59) can also describe the interimpulses evolution of the solution under the expressions

$$
\begin{aligned}
& x(t)= \Psi\left(t, t_{k}\right) x\left(t_{k}\right) \\
&+ \int_{0}^{h} \Psi\left(t, t_{k}+\tau\right) x\left(t_{k}+\tau-h\right) d \tau \\
&+\int_{0}^{t-t_{k}} \Psi\left(t, t_{k}+\tau\right) \beta\left(t_{k}+\tau\right) u\left(t_{k}+\tau\right) d \tau, \\
& \forall t \in\left[t_{k}, t_{k+1}\right),
\end{aligned}
$$

Now, assume for the sake of simplicity that the set SI of impulsive time instants is subject to the constraint $h \leq \mid t_{k+1}-$ $t_{k} \mid \leq \bar{T} ; \forall t_{k} \in$ SI and that the subsequent mixed piece-wise continuous impulsive-free and impulsive control law is used as follows:

$$
\begin{array}{r}
u(t)=g\left(t_{k}\right) \bar{g}(t) x\left(t_{k}\right)+g_{0}\left(t_{k}\right) \bar{g}_{0}(t-h) x(t-h), \\
\forall t \in\left[t_{k}, t_{k}+h\right), \quad \forall t_{k} \in \mathbf{S I}, \\
u(t)=g\left(t_{k}\right) \bar{g}(t) x\left(t_{k}\right), \quad \forall t \in\left[t_{k}+h, t_{k+1}\right), \\
\forall t_{k} \in \mathbf{S I},
\end{array}
$$

$$
\begin{array}{r}
\gamma\left(t_{k+1}\right)=\lambda\left(t_{k+1}\right) x\left(t_{k+1}^{-}\right), \\
\forall t_{k} \in \mathbf{S I},
\end{array}
$$

so that $\bar{g}(\cdot)$ and $\bar{g}_{0}$ are piecewise continuous on $\mathbf{R}_{0+}$ and $\mathbf{R}_{0+} \cup[-h, 0]$, respectively. Then, the solution of the controlled differential equation at the impulsive time instants is

$$
\begin{aligned}
& x\left(t_{k+1}\right) \\
& =\left(1+\lambda\left(t_{k+1}\right)\right) \\
& \times\left[\left(\Psi\left(t_{k+1}, t_{k}\right)+g\left(t_{k}\right)\right.\right. \\
& \times \int_{0}^{t_{k+1}-t_{k}} \Psi\left(t_{k+1}, t_{k}+\tau\right) \\
& \left.\quad \times \beta\left(t_{k}+\tau\right) \bar{g}\left(t_{k}+\tau\right) d \tau\right) x\left(t_{k}\right) \\
& +\int_{0}^{h} \Psi\left(t_{k+1}, t_{k}+\tau\right) \\
& \times\left(1+g_{0}\left(t_{k}\right) \bar{g}_{0}\left(t_{k}+\tau-h\right) \beta\left(t_{k}+\tau-h\right)\right) \\
& \left.\times x\left(t_{k}+\tau-h\right) d \tau\right] \\
& =\left(1+\lambda\left(t_{k+1}\right)\right) v\left(t_{k}, t_{k+1}\right) x\left(t_{k}\right), \\
& \forall t_{k} \in \mathrm{SI},
\end{aligned}
$$


provided that sequences $\left\{g\left(t_{k}\right)\right\}_{t_{k} \in \mathbf{S I}}$ and $\left\{g_{0}\left(t_{k}\right)\right\}_{t_{k} \in \mathbf{S I}}$ of the control law (64) are parameterized as follows:

$$
\begin{aligned}
& g\left(t_{k}\right)=\left(\nu\left(t_{k}, t_{k+1}\right)-\Psi\left(t_{k+1}, t_{k}\right)\right) \\
& \times\left(\int_{0}^{t_{k+1}-t_{k}} \Psi\left(t_{k+1}, t_{k}+\tau\right)\right. \\
&\left.\times \beta\left(t_{k}+\tau\right) \bar{g}\left(t_{k}+\tau\right) d \tau\right)^{-1}, \\
& g_{0}\left(t_{k}\right)=-\left(\int_{0}^{h} \Psi\left(t_{k+1}, t_{k}+\tau\right) x\left(t_{k}+\tau-h\right) d \tau\right) \\
& \times\left(\int_{0}^{h} \Psi\left(t_{k+1}, t_{k}+\tau\right)\right. \\
& \times\left(\bar{g}_{0}\left(t_{k}+\tau-h\right) \beta\left(t_{k}+\tau-h\right)\right) \\
&\left.\times x\left(t_{k}+\tau-h\right) d \tau\right) .
\end{aligned}
$$

Thus, Propositions 17 and 18 related to Example 15 can be applied to the sequence (65a) and (65b) and the self-mapping which generates it by replacing $e^{\int_{t_{k}}^{t_{k+1}}(\alpha(\tau)+\beta(\tau) g(\tau)) d \tau} \rightarrow$ $v\left(t_{k}, t_{k+1}\right)$. For instance, self-mapping which generates the solution sequence at impulsive time instants is asymptotically contractive if $\lim \sup _{t_{k} \in \mathbf{S I}}\left(1+\lambda\left(t_{k+1}\right)\right) \nu\left(t_{k}, t_{k+1}\right)<1$, asymptotically nonexpansive if $\lim \sup _{t_{k} \in \mathbf{S I}}\left(1+\lambda\left(t_{k+1}\right)\right) v\left(t_{k}, t_{k+1}\right) \leq$ 1 , and contractive and nonexpansive if the above conditions are replaced with similar ones at each $t_{k} \in$ SI. Similar extensions also apply to the extensions of Example 19 for a 2-semicyclic self-mapping of the delayed impulsive equation.

\section{Acknowledgments}

The authors are very grateful to the Spanish Government for its support through Grant DPI2012-30651 and to the Basque Government by its support through Grant nos. IT378-10 and SAIOTEK S-PE12UN015. They are also grateful to the University of Basque Country for its support through Grant UFI 2011/07.

\section{References}

[1] S. Saewan, P. Kanjanasamranwong, P. Kuman, and Y. J. Cho, "The modified Mann type iterative algorithm for a countable family of totally quasi-phi-asymptotically nonexpansive mappings by the hybrid generalized f-projection method," Fixed Point Theory and Applications, vol. 2013, article 63, 2013.

[2] Y. Yao, M. A. Noor, Y.-C. Liou, and S. M. Kang, "Iterative algorithms for general multivalued variational inequalities," Abstract and Applied Analysis, vol. 2012, Article ID 768272, 10 pages, 2012.

[3] M. De la Sen, "Stable iteration procedures in metric spaces which generalize a Picard-type iteration," Fixed Point Theory and Applications, vol. 2010, Article ID 953091, 15 pages, 2010.
[4] I. Inchan, "Viscosity iteration method for generalized equilibrium problems and fixed point problems of finite family of nonexpansive mappings," Applied Mathematics and Computation, vol. 219, no. 6, pp. 2949-2959, 2012.

[5] D. R. Sahu, S. M. Kang, and V. Sagar, "Approximation of common fixed points of a sequence of nearly nonexpansive mappings and solutions of variational inequality problems," Journal of Applied Mathematics, vol. 2012, Article ID 902437, 12 pages, 2012.

[6] D. R. Sahu, Z. Liu, and S. M. Kang, "Existence and approximation of fixed points of nonlinear mappings in spaces with weak uniform normal structure," Computers \& Mathematics with Applications, vol. 64, no. 4, pp. 672-685, 2012.

[7] M. Ratchagit and K. Ratchagit, "Asymptotic stability and stabilization of fixed points for iterative sequence," International Journal of Research and Reviews in Computer Science, vol. 2, no. 4, pp. 987-989, 2011.

[8] Y. J. Cho, J. K. Kim, and S. M. Kang, Fixed Point Theory and Applications, vol. 3, Nova Publishers, 2002.

[9] I. A. Rus, "Cyclic representations and fixed points," Annals of the Tiberiu Popoviciu Seminar of Functional Equations, Approximation and Convexity, vol. 3, pp. 171-178, 2005.

[10] L. B. Cirić, "Generalized contractions and fixed-point theorems," Publications de 1 Institut Mathématique, vol. 12, no. 26, pp. 19-26, 1971.

[11] A. A. Eldred and P. Veeramani, "Existence and convergence of best proximity points," Journal of Mathematical Analysis and Applications, vol. 323, no. 2, pp. 1001-1006, 2006.

[12] S. Karpagam and S. Agrawal, "Best proximity point theorems for $p$-cyclic Meir-Keeler contractions," Fixed Point Theory and Applications, vol. 2009, Article ID 197308, 9 pages, 2009.

[13] W. A. Kirk, P. S. Srinivasan, and P. Veeramani, "Fixed points for mappings satisfying cyclical contractive conditions," Fixed Point Theory, vol. 4, no. 1, pp. 79-89, 2003.

[14] E. Karapınar and I. N. Erhan, "Cyclic contractions and fixed point theory," Filomat, vol. 26, no. 4, pp. 777-782, 2012.

[15] E. Karapınar, "Best proximity points of cyclic mappings," Applied Mathematics Letters, vol. 25, no. 11, pp. 1761-1766, 2012.

[16] E. Karapınar and H. K. Nashine, "Fixed point theorem for cyclic Chatterjea type contractions," Journal of Applied Mathematics, vol. 2012, Article ID 165698, 15 pages, 2012.

[17] H. Aydi and E. Karapınar, "A fixed point result for BoydWong cyclic contractions in partial metric spaces," International Journal of Mathematics and Mathematical Sciences, vol. 2012, Article ID 597074, 11 pages, 2012.

[18] W. Sanhan, C. Mongkolkeha, and P. Kumam, "Generalized proximal $\psi$-contraction mappings and best proximity points," Abstract and Applied Analysis, vol. 2012, Article ID 896912, 19 pages, 2012.

[19] M. De la Sen, "Linking contractive self-mappings and cyclic Meir-Keeler contractions with Kannan self-mappings," Fixed Point Theory and Applications, vol. 2010, Article ID 572057, 23 pages, 2010.

[20] M. De la Sen, "On a general contractive condition for cyclic selfmappings," Journal of Applied Mathematics, vol. 2011, Article ID 542941, 17 pages, 2011.

[21] W.-S. Du, "New cone fixed point theorems for nonlinear multivalued maps with their applications," Applied Mathematics Letters, vol. 24, no. 2, pp. 172-178, 2011.

[22] S. L. Singh, S. N. Mishra, and S. Jain, "Round-off stability for multi-valued maps," Fixed Point Theory and Applications, vol. 2012, article 12, 2012. 
[23] S. L. Singh, S. N. Mishra, R. Chugh, and R. Kamal, "General common fixed point theorems and applications," Journal of Applied Mathematics, vol. 2012, Article ID 902312, 14 pages, 2012.

[24] W. Laowang and B. Panyanak, "Common fixed points for some generalized multivalued nonexpansive mappings in uniformly convex metric spaces," Fixed Point Theory and Applications, vol. 2011, article 20, 2011.

[25] H. Khandani, S. M. Vaezpour, and B. Sims, "Common fixed points of generalized multivalued contraction on complete metric spaces," Journal of Computational Analysis and Applications, vol. 13, no. 6, pp. 1025-1038, 2011.

[26] H. K. Nashine and W. Shatanawi, "Coupled common fixed point theorems for a pair of commuting mappings in partially ordered complete metric spaces," Computers \& Mathematics with Applications, vol. 62, no. 4, pp. 1984-1993, 2011.

[27] W. Shatanawi and M. Postolache, "Common fixed point results of mappings for nonlinear contraction of cyclic form in ordered metric spaces," Fixed Point Theory and Applications, vol. 2013, article 60, 2013.

[28] N. Hussain and H. K. Pathak, "Common fixed point and approximation results for $H$-operator pair with applications," Applied Mathematics and Computation, vol. 218, no. 22, pp. 11217-11225, 2012.

[29] H. K. Nashine and M. S. Khan, "An application of fixed point theorem to best approximation in locally convex space," Applied Mathematics Letters, vol. 23, no. 2, pp. 121-127, 2010.

[30] A. Latif and M. A. Kutbi, "Fixed points for $w$-contractive multimaps," International Journal of Mathematics and Mathematical Sciences, vol. 2009, Article ID 769467, 7 pages, 2009.

[31] T. Husain and A. Latif, "Fixed points of multivalued nonexpansive maps," International Journal of Mathematics and Mathematical Sciences, vol. 14, no. 3, pp. 421-430, 1991.

[32] M. S. Khan, "Common fixed point theorems for multivalued mappings," Pacific Journal of Mathematics, vol. 95, no. 2, pp. 337347, 1981.

[33] S. Reich, "Some remarks concerning contraction mappings," Canadian Mathematical Bulletin, vol. 14, pp. 121-124, 1971.

[34] I. A. Pyatyshev, "An example of boundedly approximatively compact set which is not locally compact," Russian Mathematical Surveys, vol. 62, no. 5, p. 1007, 2007.

[35] A. I. Vasilév, "The bounded compactness of sets in linear metric spaces," Mathematical Notes of the Academy of Sciences of the USSR, vol. 11, no. 6, pp. 396-401, 1972.

[36] E. Karapınar, "On best proximity points of psi-Geraghty contractions," Fixed Point Theory and Applications, vol. 2013, article 200, 2013

[37] M. Gabeleh and N. Shahzad, "Existence and convergence theorems of best proximity points," Journal of Applied Mathematics, vol. 2013, Article ID 101439, 6 pages, 2013.

[38] L.-J. Lin and W.-S. Du, "Ekeland's variational principle, minimax theorems and existence of nonconvex equilibria in complete metric spaces," Journal of Mathematical Analysis and Applications, vol. 323, no. 1, pp. 360-370, 2006.

[39] W.-S. Du, "The existence of cone critical point and common fixed point with applications," Journal of Applied Mathematics, vol. 2011, Article ID 985797, 22 pages, 2011.

[40] M. De la Sen, "Total stability properties based on fixed point theory for a class of hybrid dynamic systems," Fixed Point Theory and Applications, vol. 2009, Article ID 826438, 19 pages, 2009.
[41] T. Shen and Z. Yuan, "Stability criterion for a class of fixedpoint digital filters using two's complement arithmetic," Applied Mathematics and Computation, vol. 219, no. 9, pp. 4880-4883, 2013.

[42] H. K. Nashine, R. Pathak, P. S. Somvanshi, S. Pantelic, and P. Kumam, "Solutions for a class of nonlinear Volterra integral and integro-differential equation using cyclic $(\phi, \psi, \theta)$-contraction," Advances in Difference Equations, vol. 2013, article 106, 2013.

[43] S. Solmaz, R. Shorten, K. Wulff, and F. Ó Cairbre, "A design methodology for switched discrete time linear systems with applications to automotive roll dynamics control," Automatica, vol. 44, no. 9, pp. 2358-2363, 2008.

[44] M. De La Sen and N. Luo, "A note on the stability of linear time-delay systems with impulsive inputs," IEEE Transactions on Circuits and Systems, vol. 50, no. 1, pp. 149-152, 2003.

[45] M. De la Sen and N. Luo, "On the uniform exponential stability of a wide class of linear time-delay systems," Journal of Mathematical Analysis and Applications, vol. 289, no. 2, pp. 456-476, 2004.

[46] M. de la Sen and A. Ibeas, "On the global asymptotic stability of switched linear time-varying systems with constant point delays," Discrete Dynamics in Nature and Society, vol. 2008, Article ID 231710, 31 pages, 2008. 


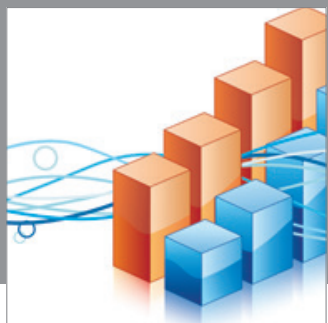

Advances in

Operations Research

mansans

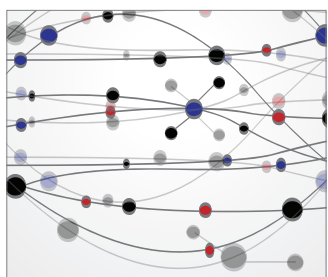

The Scientific World Journal
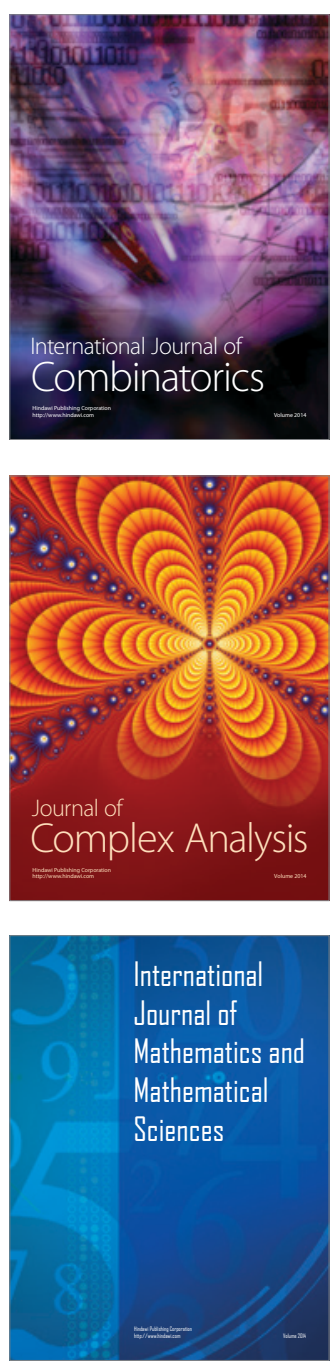
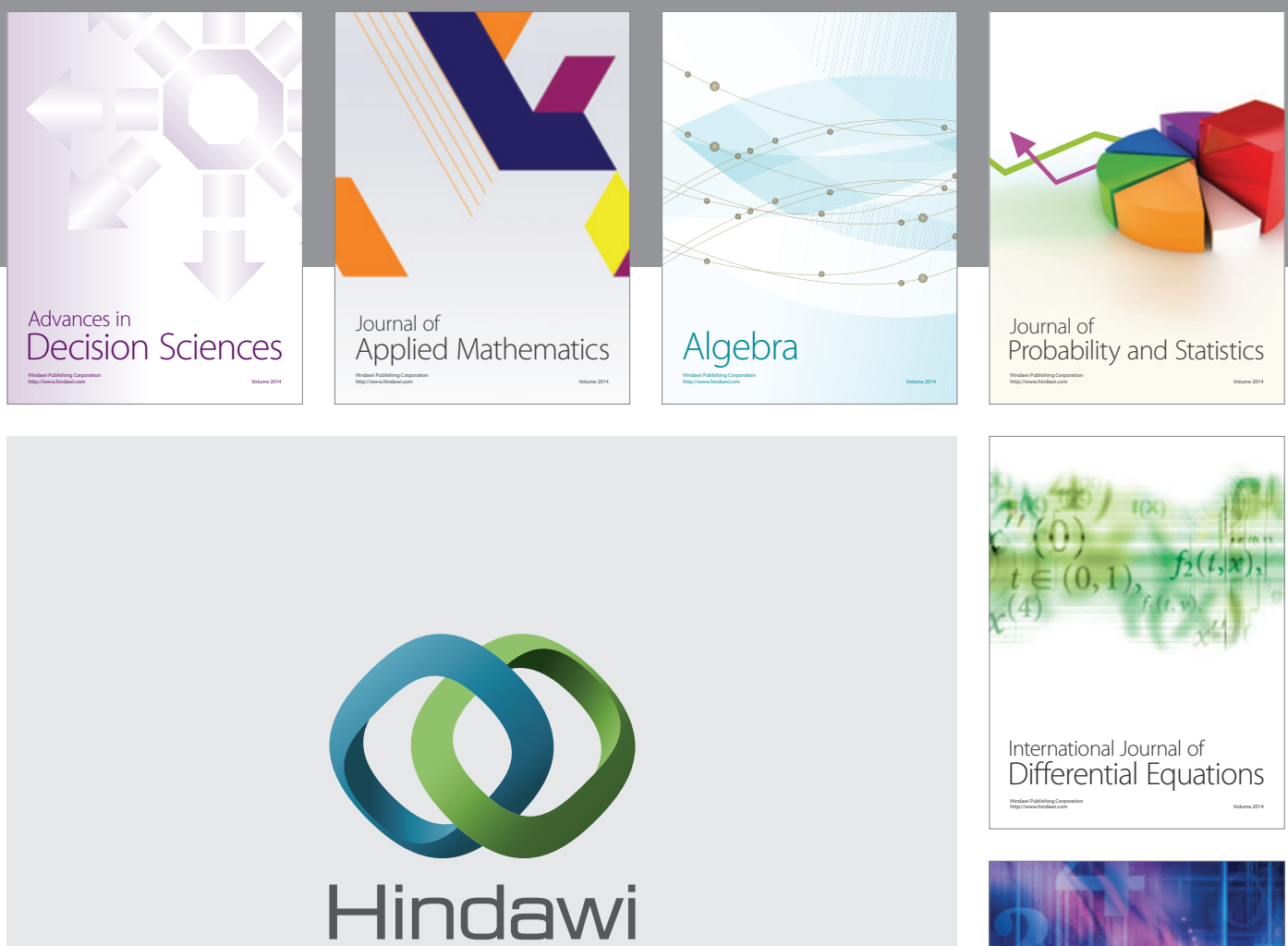

Submit your manuscripts at http://www.hindawi.com
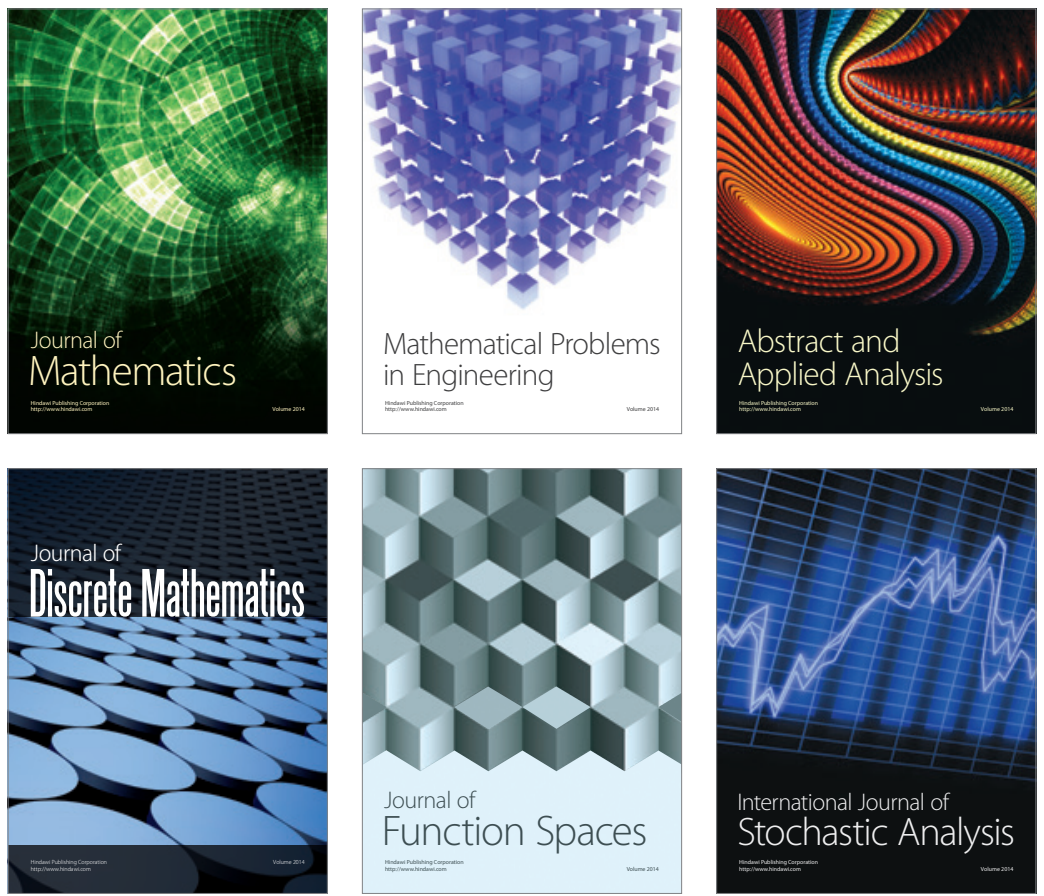

Journal of

Function Spaces

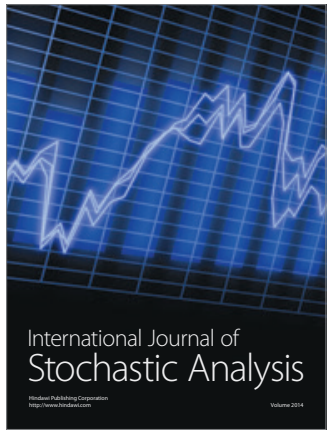

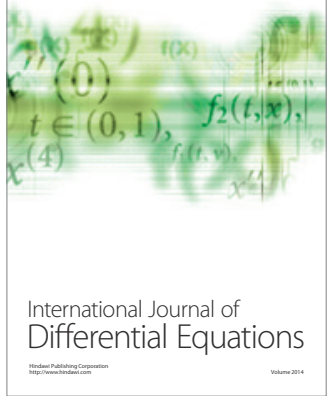
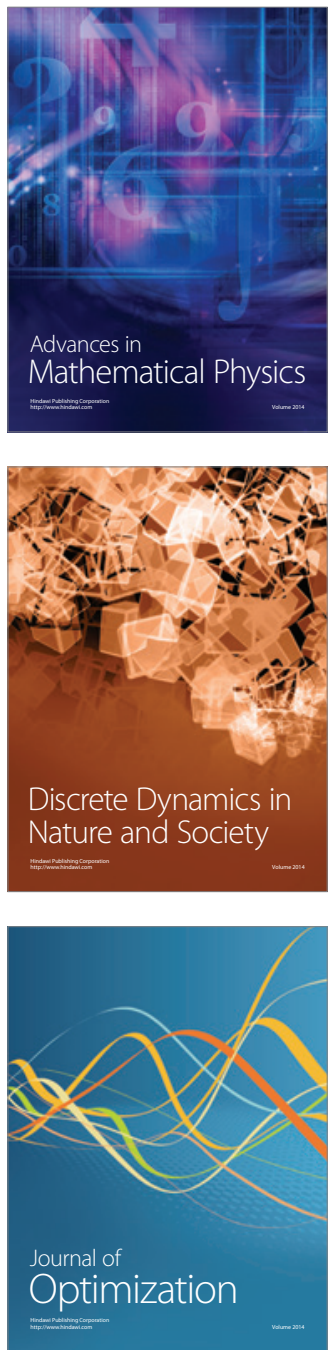